\title{
INTERSECTION NUMBERS ON THE MODULI SPACES OF STABLE MAPS IN GENUS 0
}

\author{
ALEXANDRE KABANOV AND TAKASHI KIMURA
}

\begin{abstract}
Let $V$ be a smooth, projective, convex variety. We define tautological $\psi$ and $\kappa$ classes on the moduli space of stable maps $\overline{\mathcal{M}}_{0, n}(V)$, give a (graphical) presentation for these classes in terms of boundary strata, derive differential equations for the generating functions of the Gromov-Witten invariants of $V$ twisted by these tautological classes, and prove that these intersection numbers are completely determined by the Gromov-Witten invariants of $V$. This results in families of Frobenius manifold structures on the cohomology ring of $V$ which includes the quantum cohomology as a special case.
\end{abstract}

\section{Introduction}

There has recently been a great deal of interest in $\overline{\mathcal{M}}_{g, n}(V)$, the moduli space of stable maps of genus $g$ with $n$ marked points into a smooth, projective variety $V$, an object whose construction was envisioned by Kontsevich as the proper algebro-geometric setting for Gromov-Witten invariants and quantum cohomology. The space $\overline{\mathcal{M}}_{g, n}(V)$ is a compactification of the moduli space of holomorphic maps from a genus $g$ Riemann surface with $n$ marked points to $V$ by allowing the surfaces to degenerate forming double points away from the marked points provided that a stability condition is satisfied. The moduli space of stable maps $\overline{\mathcal{M}}_{g, n}(V)$ should be regarded as a generalization of the moduli space of stable curves $\overline{\mathcal{M}}_{g, n}$, due to Deligne-Mumford, as it reduces to the latter in the special case where $V$ is a point. However, unlike the moduli space of curves, $\overline{\mathcal{M}}_{g, n}(V)$ is generally a singular space (a Deligne-Mumford stack) when the relevant obstruction bundle fails to vanish. Intersection theory on stacks requires the usage of the virtual fundamental class [5, (4) in lieu of the topological one. However, when $V$ is a smooth, projective, convex variety $\overline{\mathcal{M}}_{0, n}(V)$ are complex orbifolds [12]. We shall henceforth assume $V$ satisfies these conditions and we restrict ourselves to genus zero.

Date: May 13, 2011. 
The spaces $\overline{\mathcal{M}}_{0, n}(V)$ are equipped with evaluation morphisms ev ${ }_{i}$ : $\overline{\mathcal{M}}_{0, n}(V) \rightarrow V$ for $i=1, \ldots, n$ which evaluates the stable map on the $i^{\text {th }}$ puncture. The Gromov-Witten invariants of $V$ are the intersection numbers of the pull back of cohomology classes on $V$ via these evaluation morphisms. The Gromov-Witten invariants often solve enumerative problems in algebraic geometry, e.g. the number of rational curves in $\mathbb{C P}^{2}$ counted with suitable multiplicity [26] (see also [17, 12]). The Gromov-Witten invariants of $V$ endow $H^{\bullet}(V)$ with a deformed cup product resulting in the structure of quantum cohomology (see also [36]) which makes $H^{\bullet}(V)$ into a cohomological field theory (CohFT) [26, 30] (or equivalently, a (formal) Frobenius manifold [8, 16, 30]). The Gromov-Witten invariants can be encoded in a generating function (called the potential) on $H^{\bullet}(V)$ which must obey the WDVV (WittenDijkgraaf-Verlinde-Verlinde) equations. The WDVV equations allows the recursive computation of the Gromov-Witten invariants of certain homogeneous spaces, e.g. projective spaces, Grassmannians, etc. 26, 12, 17]

Another set of cohomology classes on $\overline{\mathcal{M}}_{0, n}(V)$ are those associated to its universal curve. The tautological $\psi$ classes are the first Chern classes of tautological line bundles on $\overline{\mathcal{M}}_{0, n}(V)$. Certain intersection numbers of the $\psi$ classes and the pull back of cohomology classes on $V$ via the evaluation morphisms are the called Gromov-Witten invariants twisted by the $\psi$ classes. These twisted invariants are of great interest as the associated potential is conjectured to obey a highest weight condition for the Virasoro Lie algebra [9, 10], a conjecture which has some intriguing evidence behind it [2, 34]. This conjecture has been proven in the case where $V$ is a point and is a consequence of Kontsevich's proof of the Witten conjecture [29, 25, 7]. There, the highest weight condition gives rise to recursion relations which completely determine these intersection numbers in all genera.

In this paper, we begin by exploiting the canonical stratification of $\overline{\mathcal{M}}_{0, n}(V)$ by complex orbifolds which generalize the stratification of $\overline{\mathcal{M}}_{0, n}$. These strata are indexed by decorated trees which can be used to develop a graphical language describing cohomology classes on $\overline{\mathcal{M}}_{0, n}(V)$. This graphical language is particularly useful in describing the behavior of these classes under restriction to the strata and under the push forward and pull back with respect to the forgetful map $\overline{\mathcal{M}}_{0, n+1}(V) \rightarrow \overline{\mathcal{M}}_{0, n}(V)$.

We then introduce the tautological $\psi$ and $\kappa$ classes on $\overline{\mathcal{M}}_{0, n}(V)$ generalizing those classes from $\overline{\mathcal{M}}_{0, n}$. The definition of the $\kappa$ classes is new and has the novel feature that their proper definition involves the pull 
backs via the evaluation morphisms of the cohomology classes on $V$. We prove restriction properties for these classes to the boundary strata and derive a (graphical) presentation of the $\psi$ and $\kappa$ classes in terms of boundary strata generalizing the presentation for the same classes in [18] on the moduli space of curves.

We prove that the Gromov-Witten classes twisted by the $\psi$ and $\kappa$ classes endows $H^{\bullet}(V)$ with a family of CohFT structures by pushing them forward to the moduli space of curves. These CohFT structures is a deformation of the cup product containing, as a special case, the product on quantum cohomology.

Finally, we prove recursion relations for the intersection numbers of the Gromov-Witten invariants twisted by the $\psi$ and $\kappa$ classes by using these presentations and the restriction properties of these cohomology classes. These recursion relations can be encoded in terms of a potential for these intersection numbers satisfying a system of differential equations called the topological recursion relations. We also prove two other equations, the analogs of the puncture and dilaton equations, which do not use the presentation of the $\psi$ and $\kappa$ classes and show that all of these intersection numbers are completely determined by the Gromov-Witten invariants of $V$.

Consider the special case where there are no $\kappa$ classes. These topological recursion relations were originally written down by Witten 37 in the context of topological gravity coupled to topological matter before the moduli space of stable maps were even defined! A proof of Witten's equations has been announced in [27] to appear in [31. Some work toward this direction has also been done in [35]. (In the setting of symplectic geometry, these equations were proven in [36].) The proof of the puncture and dilaton equations for the $\psi$ classes is also forthcoming in [31].

Our work is a generalization of these results to the case where the $\kappa$ classes are included. The $\kappa$ classes are important here because their restriction properties manifestly yield families of CohFT structures on $H^{\bullet}(V)$ and provide the necessary cohomology classes (with values in the tensor algebra of $\left.H^{\bullet}(V)\right)$ on $\overline{\mathcal{M}}_{0, n}(V)$ which define the CohFT. This is in marked contrast to the case involving only the $\psi$ classes where the tensor algebra-valued forms are not manifest (see 18, 28, 21 for a special case).

Furthermore, both the Gromov-Witten invariants twisted by only the $\kappa$ classes and the same twisted by only the $\psi$ classes give rise to families of genus zero CohFT structures on $H^{\bullet}(V)$. The families corresponding to the $\kappa$ classes and those associated to the $\psi$ classes are, in some sense, 
dual to each other and this correspondence extends to higher genera [19.

The paper is structured as follows. In the first section, we review the geometry of the moduli space of genus 0 stable maps $\overline{\mathcal{M}}_{0, n}(V)$ with $n$ marked points associated to a smooth, projective, convex variety $V$. In the second section, we develop a graphical calculus to describe cohomology classes on $\overline{\mathcal{M}}_{0, n}(V)$. In the third section, we introduce the tautological $\psi$ and $\kappa$ classes on $\overline{\mathcal{M}}_{0, n}(V)$. We prove restriction properties and lifting properties of these classes and obtain a graphical presentation of these classes in terms of boundary strata. In the fourth section, we show that these intersection numbers endow the cohomology ring $H^{\bullet}(V)$ with families of CohFT structures. In the fifth section, we derive differential equations satisfied by the potentials for these intersection numbers and prove that they are completely determined by the Gromov-Witten invariants.

Acknowledgment. We are greatful to the Max Planck Institut für Mathematik, where this work was initiated, for their financial support and for providing a wonderfully stimulating atmosphere. We would like to thank D. Abramovich, P. Belorousski, E. Getzler, Yu. Manin, R. Pandharipande, and D. Zagier for useful conversations. We would like to continue to thank K. Belabas for his $\mathrm{T}_{\mathrm{E} X n i c a l}$ assistance and for providing the music.

\section{The Moduli Spaces of Stable Maps}

In this section, we recall properties of the moduli space of stable maps whose intersection numbers are the main object of study in this paper. These spaces can be viewed as a generalization of the moduli spaces of stable curves due to Deligne-Mumford, a canonical compactification of the moduli space of configurations of $n$ labeled, points on Riemann surfaces of genus $g$.

To describe the moduli space of stable maps, one needs to generalize the notion of a stable curve.

Definition 1.1. A quasi-stable curve of genus $g$ with $n$ marked points, denoted by $\left(C ; x_{1}, x_{2}, \ldots, x_{n}\right)$, is a connected, complex projective, reduced, curve with (at most) double points of arithmetic genus $g$ together with distinct points $x_{1}, x_{2}, \ldots, x_{n}$ in $C$ away from the double points. A special point of $C$ is either a marked point or a double point.

Definition 1.2. Let $V$ be a smooth, projective variety and $\beta$ in $H_{2}(V, \mathbb{Z})$. The moduli space of stable maps $\overline{\mathcal{M}}_{g, n}(V, \beta):=\left\{\left[C, f ; x_{1}, x_{2}, \ldots, x_{n}\right]\right\}$ consists of isomorphism classes of the following data: a quasi-stable 
curve of genus $g$ with $n$ marked points $\left(C ; x_{1}, x_{2}, \ldots, x_{n}\right)$ together with a morphism $f: C \rightarrow V$ such that $\beta=[f(C)]$ satisfying the stability condition that each irreducible component which is mapped to a point in $V$ musts be stable in the usual sense, i.e. each irreducible component minus its special points must have negative Euler characteristic.

Not all $\beta \in H_{2}(V, \mathbb{Z})$ are represented by such curves in $V$. Let $B(V)$ be the semi-subgroup of $H_{2}(V, \mathbb{Z})$ generated by the images of the curves in $V$.

Definition 1.3. The evaluation morphism associated to the $i^{\text {th }}$ marked point $e v_{i}: \overline{\mathcal{M}}_{g, n}(V, \beta) \rightarrow V$ takes the point $\left[C, f ; x_{1}, x_{2}, \ldots, x_{n}\right]$ to $f\left(x_{i}\right)$ for all $i=1, \ldots, n$. The stabilization morphism st $: \overline{\mathcal{M}}_{g, n}(V, \beta) \rightarrow$ $\overline{\mathcal{M}}_{g, n}$, defined when $2 g-2+n>0$, takes the point $\left[C, f ; x_{1}, x_{2}, \ldots, x_{n}\right]$ to $\left[C ; x_{1}, x_{2}, \ldots, x_{n}\right]^{\text {st }}$. The latter means that one contracts all components that became unstable after forgetting $f$. Let $\pi: \overline{\mathcal{M}}_{g, n+1}(V, \beta) \rightarrow$ $\overline{\mathcal{M}}_{g, n}(V, \beta)$ be the morphism which forgets the $i^{\text {th }}$ point followed by stabilization, i.e. $\left[C, f ; x_{1}, x_{2}, \ldots, x_{n+1}\right] \mapsto\left[C, f ; x_{1}, \ldots, \widehat{x}_{i}, \ldots, x_{n+1}\right]^{\mathrm{st}}$. This morphism is the universal curve over $\overline{\mathcal{M}}_{g, n}(V, \beta)$ [3] 5 ].

Theorem 1.4. [1], [12] Let $V$ be a smooth, projective variety and $\beta$ in $\mathrm{H}_{2}(V, \mathbb{Z})$. The moduli spaces $\overline{\mathcal{M}}_{g, n}(V, \beta)$ exist and are complete, Deligne-Mumford stacks.

The moduli space of stable maps becomes the moduli space of curves by choosing the variety $V$ to be a point. The moduli space of curves $\overline{\mathcal{M}}_{g, n}$ is a smooth stack (orbifolds in the algebraic category) for all genera. In general, $\overline{\mathcal{M}}_{g, n}(V, \beta)$ is highly singular. A useful criterion by which one can obtain nice moduli spaces, at least in genus zero, is that $V$ be convex.

Definition 1.5. A smooth, projective variety $V$ is said to be convex if for all morphisms $f: \mathbb{C P}^{1} \rightarrow V$, the cohomology $H^{1}\left(\mathbb{C P}^{1}, f^{*} T_{V}\right)$ vanishes where $T_{V}$ is the (holomorphic) tangent bundle.

Theorem 1.6. [5], [12] Let $V$ be a smooth, projective, convex variety of dimension $D$ then the space $\overline{\mathcal{M}}_{0, n}(V, \beta)$ is a locally normal, projective variety of pure (complex) dimension $D+n-3+\int_{\beta} c_{1}\left(T_{V}\right)$ and can have, at worst, orbifold singularities. In addition, the divisor corresponding to the locus of the singular curves is a normal crossing divisor up to a quotient by a finite group.

Definition 1.7. Let $V$ be a smooth, projective, convex variety then the (genus zero) Gromov-Witten invariants of $V$ are maps $\left(H^{\bullet}(V)\right)^{\otimes n} \rightarrow$ 
$\mathbb{C}$ given by

$$
\gamma_{1} \otimes \gamma_{2} \otimes \ldots \otimes \gamma_{n} \mapsto\left\langle\mathrm{ev}_{1}^{*} \gamma_{1} \mathrm{ev}_{2}^{*} \gamma_{2} \ldots \mathrm{ev}_{n}^{*} \gamma_{n}\right\rangle_{\beta}
$$

where

$$
\left\langle\mathrm{ev}_{1}^{*} \gamma_{1} \mathrm{ev}_{2}^{*} \gamma_{2} \ldots \mathrm{ev}_{n}^{*} \gamma_{n}\right\rangle_{\beta}:=\int_{\overline{\mathcal{M}}_{0, n}(V, \beta)} \mathrm{ev}_{1}^{*} \gamma_{1} \mathrm{ev}_{2}^{*} \gamma_{2} \ldots \mathrm{ev}_{n}^{*} \gamma_{n}
$$

where $\gamma_{1}, \gamma_{2}, \ldots, \gamma_{n}$ are elements in $H^{\bullet}(V), n$ is the number of marked points, $\beta$ belongs to $B(V)$, and the product is understood to be the cup product.

Gromov-Witten invariants can also be defined in higher genera and for certain nonconvex varieties provided that one uses the proper notion of fundamental class (the so-called virtual fundamental class [4], [5]) of the moduli spaces of stable maps.

The moduli space $\overline{\mathcal{M}}_{0, n}(V, \beta)$ for $V$ a smooth, projective, convex variety is endowed with a canonical stratification by strata which are indexed by certain decorated trees called stable trees.

Let us introduce some notation regarding graphs (and trees). All graphs we shall consider are connected and have vertices with a valence greater than or equal to zero. Given such a tree $\Gamma$, let $V(\Gamma)$ be its set of vertices, $E(\Gamma)$ be its set of edges, and $S(\Gamma)$ be its set of tails. Each edge has two endpoints belonging to $V(\Gamma)$ which are allowed to be the same. Each tail has only one endpoint. For all $v \in V(\Gamma)$, let $n(v)$ be the number of half-edges emanating from $v$. Each edge gives rise to two half-edges, and each tail to one half-edge.

A stable graph consists of a quadruple $(\Gamma, g, \beta, \mu)$, where $\Gamma$ is a connected graph as above, $g: V(\Gamma) \rightarrow \mathbb{Z}_{\geq 0}, \beta: V(\Gamma) \rightarrow B(V)$, and $\mu$ is a bijection between $S(\Gamma)$ and a given set $I$. We also require that for each vertex $v$ the stability condition be satisfied: if $\beta(v)=0$, then $2 g(v)-2+n(v)>0$. We define the genus, $g(\Gamma)$, of $\Gamma$ to be $b_{1}(\Gamma)+\sum_{v \in V(\Gamma)} g(v)$, where $b_{1}$ is the first Betti number. We also define the degree, $\beta(\Gamma)$, of $\Gamma$ to be $\sum_{v \in V(\Gamma)} \beta(v)$. A stable tree is a stable graph of genus zero.

To each stable map $\left[C, f ; x_{1}, \ldots, x_{n}\right] \in \overline{\mathcal{M}}_{0, n}(V, \beta)$ one can associate a stable tree, called its dual tree, by collapsing each irreducible component to a point forming a vertex, drawing a half-edge emanating from this vertex for each special point on that irreducible component and connecting any two half-edges if the the two components share a double point, and then decorating each vertex $v$ with the arithmetic genus $g(v)$ of its corresponding irreducible component and with $\beta(v)$ which is the image of fundamental class of that irreducible component in $B(V)$. 
Finally, the tails are decorated with integers $\{1, \ldots, n\}$ corresponding to the marked points on $C$.

In this way each stable tree $\Gamma$ with $n$ tails of genus 0 and degree $\beta$ determines a closed sub-orbifold $\overline{\mathcal{M}}_{\Gamma}$ of $\overline{\mathcal{M}}_{0, n}(V, \beta)$ by taking the closure of the locus of stable maps whose dual tree is $\Gamma$ [12]. Each $\overline{\mathcal{M}}_{\Gamma}$ is a smooth stack (orbifold) of complex codimension $|E(\Gamma)|$ in $\overline{\mathcal{M}}_{0, n}(V, \beta)$. We denote the orbifold fundamental class of $\overline{\mathcal{M}}_{\Gamma}$ by $\left[\overline{\mathcal{M}}_{\Gamma}\right]$. According to [24], the stratification defined above is finite.

The stable trees $\Gamma$ play a further role since it indicates how each stratum $\overline{\mathcal{M}}_{\Gamma}$ can be expressed as a fibered product of fundamental classes of the moduli spaces quotiented by the action of the automorphism group of the stable tree. More precisely, if $v \in V(\Gamma)$ then we denote by $\overline{\mathcal{M}}(v)$ the moduli space $\overline{\mathcal{M}}_{0, n(v)}(V, \beta(v))$. Each edge of $\Gamma$ joining vertices $v_{1}$ and $v_{2}$ determines evaluation morphisms $\overline{\mathcal{M}}\left(v_{1}\right) \rightarrow V$ and $\overline{\mathcal{M}}\left(v_{2}\right) \rightarrow V$. The stratum $\overline{\mathcal{M}}_{\Gamma}$ is isomorphic as a stack (orbifold) to the fibered product of $\overline{\mathcal{M}}(v), v \in V(\Gamma)$ over $V^{|E(\Gamma)|}$ with respect to the evaluation morphisms corresponding to each edge quotiented by the obvious action of $\operatorname{Aut}(\Gamma)$, the automorphism group of $\Gamma$. We denote by $\widehat{\prod}_{v \in V(\Gamma)} \overline{\mathcal{M}}(v)$ the fibered product defined above, by $\rho_{\Gamma}$ the composition

$$
\widehat{\prod}_{v \in V(\Gamma)} \overline{\mathcal{M}}(v) \rightarrow \overline{\mathcal{M}}_{\Gamma} \rightarrow \overline{\mathcal{M}}_{0, n}(V, \beta)
$$

and by $j_{\Gamma}$ the inclusion

$$
\widehat{\prod_{v \in V(\Gamma)}} \overline{\mathcal{M}}(v) \rightarrow \prod_{v \in V(\Gamma)} \overline{\mathcal{M}}(v)
$$

\section{Presentation of Cohomology Classes via Graphs}

In this section we introduce a graphical notation for certain cohomology classes on the moduli spaces of stable maps which uses the graphical presentation of the boundary strata. Here we generalize the notation introduced in [18, 2.2].

Throughout the rest of this paper we assume that $g=0$ and that $V$ is a convex, smooth, projective variety.

We start with explaining how to push forward and pull back the cohomology classes represented by graphs under the universal curve morphism. Let $\pi: \overline{\mathcal{M}}_{0, n+1}(V, \beta) \rightarrow \overline{\mathcal{M}}_{0, n}(V, \beta)$ be defined by forgetting the $i^{\text {th }}$ marked point. First we explain the push forward. Suppose that $\Gamma^{\prime}$ represents a stratum in $\overline{\mathcal{M}}_{0, n+1}(V, \beta)$. If removing the tail labeled by $i$ from $\Gamma^{\prime}$ does not destabilize it, then $\pi_{*}\left(\left[\overline{\mathcal{M}}_{\Gamma}^{\prime}\right]\right)=0$. On the other 
hand, if removing the tail labeled $i$ does destabilize $\Gamma^{\prime}$, then

$$
\pi_{*}\left(\left[\overline{\mathcal{M}}_{\Gamma^{\prime}}\right]\right)=\frac{|\operatorname{Aut} \Gamma|}{\left|\operatorname{Aut} \Gamma^{\prime}\right|}\left[\overline{\mathcal{M}}_{\Gamma}\right],
$$

where $\Gamma$ is obtained by stabilization [32].

Next we explain how to pull back the cohomology classes represented by graphs. Let $\overline{\mathcal{M}}_{\Gamma}$ be a stratum in $\overline{\mathcal{M}}_{0, n}(V, \beta)$. The pull back of its fundamental class is a subvariety of $\overline{\mathcal{M}}_{0, n+1}(V, \beta)$ corresponding to the sum of $|V(\Gamma)|$ graphs each of which is obtained by attaching a tail numbered $i$ to a vertex of $\Gamma$ [32].

These rules for pushing forward and pulling back cohomology classes on the moduli space of stable maps are analogous to the rules for the moduli space of curves.

In the sequel we will need trees whose vertices are decorated with more than its degree. In addition, we will decorate the vertices with cohomology classes. Let $\Gamma$ be a stable tree that determines a subvariety of $\overline{\mathcal{M}}_{0, n}(V, \beta)$, and let $\gamma_{v} \in H^{\bullet}(\overline{\mathcal{M}}(v)), v \in V(\Gamma)$. We denote the cohomology class

$$
\frac{1}{|\operatorname{Aut} \Gamma|} \rho_{\Gamma *} j_{\Gamma}^{*}\left(\otimes_{v} \gamma_{v}\right)
$$

by the picture of $\Gamma$ where each vertex $v$ is in addition labeled by the cohomology class $\gamma_{v}$. We omit this label if $\gamma_{v}=1$, i.e., $\gamma_{v}$ is the orbifold fundamental class of $\overline{\mathcal{M}}(v)$. In particular, $\left[\overline{\mathcal{M}}_{\Gamma}\right]$ is represented by $\Gamma$ with no additional labels attached.

Before we prove the lemma below that describes the push forward of the cohomology classes represented by decorated stable trees we would like to recall some properties of the fibered products. Let $X$ and $Y$ be two smooth varieties or orbifolds with morphisms $X \rightarrow V$, $Y \rightarrow V$ to a smooth variety $V$. Then the fibered product $X \times_{V} Y$ is a subspace of $X \times Y$, and we denote the corresponding inclusion by $j$. The cohomology $H^{\bullet}\left(X \times_{V} Y\right)$ is isomorphic to $H^{\bullet}(X) \otimes_{H^{\bullet}(V)} H^{\bullet}(Y)$, and the restriction $j^{*}$ is surjective and the push forward $j_{*}$ is injective. The composition $j_{*} j^{*}(\alpha)$ is equal to $\Delta \alpha$, where $\alpha \in H^{\bullet}(X \times Y)$, and $\Delta=j_{*}(1)$ is the Thom class. It is the pull back of the Thom class of $V$ in $V \times V$.

One can easily generalize the discussion above to the fibered products of more than two spaces. If $j$ denotes the inclusion of the fibered product into the direct product, then $j^{*}$ is surjective, $j_{*}$ is injective, and the composition $j_{*} j^{*}$ is the multiplication by the pull back of the Thom class of $V^{k}$ in $V^{n}$, where the inclusion of $V^{k}$ into $V^{n}$ is determined by the fibered product. 
Lemma 2.1. Let $\pi: \overline{\mathcal{M}}_{0, n+1}(V, \beta) \rightarrow \overline{\mathcal{M}}_{0, n}(V, \beta)$ be the universal curve determined by forgetting the tail labeled $i$. Let $\Gamma^{\prime}$ be a tree that determines a subvariety in $\overline{\mathcal{M}}_{0, n+1}(V, \beta)$ and $\Gamma$ be the tree obtained from $\Gamma^{\prime}$ by removing the tail labeled $i$. We assume that removing the tail labeled $i$ does not destabilize $\Gamma^{\prime}$, and for each vertex $v^{\prime}$ of $\Gamma^{\prime}$ we denote by $v$ the corresponding vertex of $\Gamma$. Let $w^{\prime}$ be the vertex from which the tail numbered $i$ emanates, and let $\pi(w): \overline{\mathcal{M}}\left(w^{\prime}\right) \rightarrow \overline{\mathcal{M}}(w)$ be the universal curve over $\overline{\mathcal{M}}(w)$ which forgets $i$.

If $\gamma^{\prime} \in H^{\bullet}\left(\overline{\mathcal{M}}_{0, n+1}(V, \beta)\right)$ is represented by $\Gamma^{\prime}$ decorated with the cohomology classes $\gamma_{v^{\prime}}^{\prime}, v^{\prime} \in V\left(\Gamma^{\prime}\right)$, then its push forward under $\pi$ is the cohomology class $\gamma$ represented by the multiple $\frac{\mid \text { Aut } \Gamma \mid}{\left|\operatorname{Aut} \Gamma^{\prime}\right|}$ of $\Gamma$ decorated with the cohomology classes $\gamma_{v}$, where $\gamma_{v}=\gamma_{v^{\prime}}^{\prime}$ when $v \neq w$, and $\gamma_{w}=$ $\pi(w)_{*}\left(\gamma_{w^{\prime}}\right)$.

Proof. For each vertex $v^{\prime}$ of $\Gamma^{\prime}$ there exists the corresponding vertex $v$ of $\Gamma$, and $\overline{\mathcal{M}}\left(v^{\prime}\right)$ is isomorphic to $\overline{\mathcal{M}}(v)$. These isomorphisms can be chosen so that they respect the fibered products. We denote by $X$ the product $\prod_{v \neq w} \overline{\mathcal{M}}(v)$ and by $X_{V}$ the fibered product $\widehat{\prod}_{v \neq w} \overline{\mathcal{M}}(v)$. Consider the following commutative diagram where the horizontal arrows are natural morphisms, and two left vertical morphisms are induced by $\pi(w)$ :

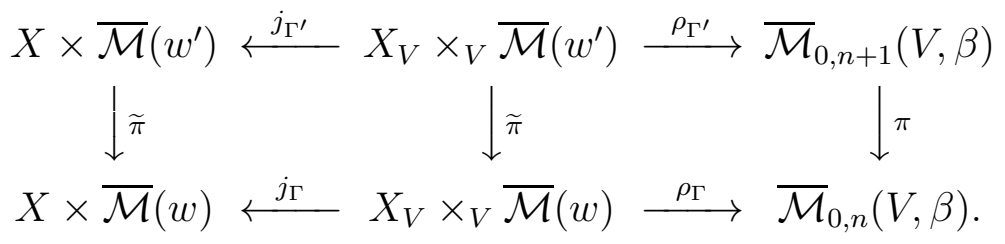

In order to prove the statement of the lemma one needs to show that

$$
\pi_{*} \rho_{\Gamma^{\prime} *} j_{\Gamma^{\prime}}^{*}=\rho_{\Gamma *} j_{\Gamma}^{*} \widetilde{\pi}_{*} .
$$

This reduces to showing that $\widetilde{\pi}_{*} j_{\Gamma^{\prime}}^{*}=j_{\Gamma}^{*} \widetilde{\pi}_{*}$. The latter follows from [11, 6.2].

Remark . If in the lemma above one assumes that $\Gamma^{\prime}$ destabilizes after forgetting the tail labeled by $i$, then $\overline{\mathcal{M}}\left(w^{\prime}\right)$ is isomorphic to a point. If the degree of $\gamma_{w^{\prime}}$ is greater than 0 attached to $w^{\prime}$, then the decorated graph represents 0 in cohomology. If $\gamma_{w^{\prime}}=1$, then one pushes the corresponding cohomology class down as in the case without decorations - by stabilizing and dividing by the orders of the appropriate automorphism groups. 
For example, consider the cohomology class $\gamma$ in $\overline{\mathcal{M}}_{0,5}(V, \beta)$ (where $\beta$ is assumed to be nonzero) represented by the decorated stable tree

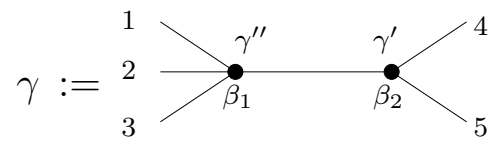

where it is understood that $\beta_{1}+\beta_{2}=\beta$ and $\gamma^{\prime}$ is a cohomology class on the space $\overline{\mathcal{M}}_{0,3}\left(V, \beta_{2}\right)$ associated to the right vertex and $\gamma^{\prime \prime}$ is a cohomology class on the space $\overline{\mathcal{M}}_{0,4}\left(V, \beta_{1}\right)$ associated to the left vertex. Consider the morphism $\pi: \overline{\mathcal{M}}_{0,5}(V, \beta) \rightarrow \overline{\mathcal{M}}_{0,4}(V, \beta)$ which forgets the $5^{\text {th }}$ marked point. If $\beta_{2}$ is nonzero then

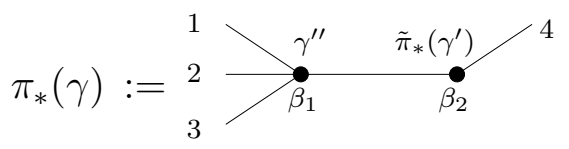

where $\tilde{\pi}: \overline{\mathcal{M}}_{0,3}\left(V, \beta_{2}\right) \rightarrow \overline{\mathcal{M}}_{0,2}\left(V, \beta_{2}\right)$ is the projection forgetting the point labeled by 5 associated to the right vertex. On the other hand, if $\beta_{2}=0$ then the right hand side of the previous equation is now unstable. If $\gamma^{\prime}$ has nonzero dimension then $\pi_{*}(\gamma)$ vanishes. If $\gamma^{\prime}$ is the unit element then

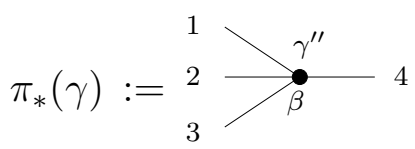

In all of these cases, the automorphism group is trivial so the prefactor does not arise.

\section{Tautological Classes}

In this section we introduce the tautological $\psi$ and $\kappa$ classes on the moduli spaces of stable maps which generalize the corresponding tautological classes on the moduli spaces of stable curves. We will also discuss the properties of the pull back of the $\psi$ classes under the stabilization morphism, and derive the restriction properties of the tautological classes to the boundary strata.

Let $\pi: \overline{\mathcal{M}}_{0, n+1}(V, \beta) \rightarrow \overline{\mathcal{M}}_{0, n}(V, \beta)$ be the universal curve. We assume that $\pi$ "forgets" the $(n+1)^{\text {st }}$ marked point. The morphism $\pi$ has $n$ canonical sections $\sigma_{1}, \ldots, \sigma_{n}$. We denote their images by $D_{1}, \ldots, D_{n}$. If $g=0$ and $V$ is convex each $D_{i}$ is a divisor. We denote by $\omega$ the relative dualizing sheaf of $\pi$. It is an invertible sheaf, that is, it determines a line bundle on $\overline{\mathcal{M}}_{0, n+1}(V, \beta)$ [5]. 
Definition 3.1. For each $i=1, \ldots, n$ the tautological line bundle $\mathcal{L}_{i}$ on $\overline{\mathcal{M}}_{0, n}(V, \beta)$ is $\sigma_{i}^{*} \omega$. The tautological class $\psi_{i} \in H^{2}\left(\overline{\mathcal{M}}_{0, n}(V, \beta)\right)$ is equal to the first Chern class $c_{1}\left(\mathcal{L}_{i}\right)$.

One can also pull back cohomology classes from $V$ to $\overline{\mathcal{M}}_{0, n}(V, \beta)$ using the evaluation maps. The definition of the $\kappa$ classes involves both, powers of the $\psi$ classes and these pull backs.

Definition 3.2. The tautological class $\kappa_{a}$ in $H^{\bullet}\left(\overline{\mathcal{M}}_{0, n}(V, \beta)\right) \otimes H^{\bullet}(V)^{*}$ for $a \geq-1$ is defined as follows. For each $\gamma \in H^{\bullet}(V)$, the cohomology class $\kappa_{a}(\gamma)$ is the push forward of $\psi_{n+1}^{a+1} e v_{n+1}^{*}(\gamma)$ with respect to the projection $\pi: \overline{\mathcal{M}}_{0, n+1}(V, \beta) \rightarrow \overline{\mathcal{M}}_{0, n}(V, \beta)$ which forgets the $(n+1)^{\text {st }}$ marked point. In particular, if $\gamma$ has definite degree $|\gamma|$ then $\kappa_{a}(\gamma)$ has degree $2 a+|\gamma|$. If $\left\{e_{\alpha}\right\}_{\alpha \in A}$, is a homogeneous basis for $H^{\bullet}(V)$ such that $e_{0}$ is the identity element then $\kappa_{a, \alpha}$ denotes the cohomology class $\kappa_{a}\left(e_{\alpha}\right)$.

Remark. The class $\kappa_{-1,0}$ vanishes due to dimensional reasons. In addition, all classes $\kappa_{-1}(\gamma)$ vanish on $\overline{\mathcal{M}}_{0, n}(V, 0)$.

Our definition corresponds to the "modified" $\kappa$ classes defined by Arbarello and Cornalba [1] rather than the "classical" $\kappa$ classes defined by Mumford [32]. It is easy to see that $\psi_{n+1}=c_{1}\left(\omega\left(D_{1}+\ldots+D_{n}\right)\right)$, and therefore one can also define the $\kappa$ classes using the relative dualizing sheaf.

Next we will show that the $\psi$ classes restrict to the strata in the expected manner. For a more general treatment, we again refer to 31] (see also [33]).

Lemma 3.3. Let $\Gamma$ be a stable tree which determines a stratum in $\overline{\mathcal{M}}_{0, n}(V, \beta)$. Suppose that the tail labeled $i$ is attached to $w \in V(\Gamma)$. Denote the class $\psi_{i}$ on $\overline{\mathcal{M}}_{0, n}(V, \beta)$ by $\psi_{i}$, and on $\overline{\mathcal{M}}(w)$ by $\psi_{i}^{\prime}$. Then $\rho_{\Gamma}^{*}\left(\psi_{i}\right)=j_{\Gamma}^{*} \psi_{i}^{\prime}$.

Proof. Let $\pi: \overline{\mathcal{M}}_{0, n+1}(V, \beta) \rightarrow \overline{\mathcal{M}}_{0, n}(V, \beta)$ and $\pi(w): \overline{\mathcal{M}}\left(w^{\prime}\right) \rightarrow \overline{\mathcal{M}}(w)$ be the universal curves forgetting the marked point labeled $n+1$, and $\sigma_{i}$ be the corresponding sections which we denote by the same symbol. We use the same notation as in the proof of Lem. 2.1. Consider the commutative diagram

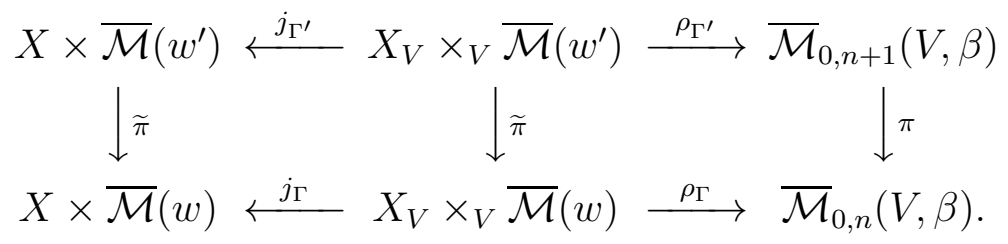


Let us denote by $\omega$ with a subscript the relative dualizing sheaf of the morphism corresponding to the subscript. It is clear that $\rho_{1}^{*} \omega_{\pi}=$ $\omega_{1 \times_{V} \widetilde{\pi}}=j_{1}^{*} \omega_{1 \times \widetilde{\pi}}$ when restricted to the image of $\sigma_{i}$. It follows that

$$
\rho_{\Gamma}^{*} \psi_{i}=\sigma_{i}^{*} \rho_{\Gamma^{\prime}}^{*}\left(\omega_{\pi}\right)=\sigma_{i}^{*} j_{\Gamma^{\prime}}^{*}\left(\omega_{\tilde{\pi}}\right)=j_{\Gamma}^{*} \psi_{i}^{\prime} .
$$

It is easy to verify that the pull backs of the cohomology classes on $V$ under the evaluation maps restrict in the same manner.

Lemma 3.4. Let $\Gamma$ be a stable tree which determines a stratum in $\overline{\mathcal{M}}_{0, n}(V, \beta)$. Denote the class $\kappa_{a, \alpha}$ on $\overline{\mathcal{M}}_{0, n}(V, \beta)$ (resp. $\overline{\mathcal{M}}(v)$, where $v \in V(\Gamma))$ by $\kappa($ resp. $\kappa(v))$. Then

$$
\rho_{\Gamma}^{*}(\kappa)=j_{\Gamma}^{*} \sum_{v \in V(\Gamma)} \kappa(v) .
$$

Proof. Consider the set $\mathcal{G}$ of cardinality $|V(\Gamma)|$ whose elements are graphs $\Gamma^{\prime}$ each of which is obtained from $\Gamma$ by attaching a tail labeled $n+1$ to one of the vertices of $\Gamma$. Each $\Gamma^{\prime} \in \mathcal{G}$ determines a stratum of $\overline{\mathcal{M}}_{0, n+1}(V, \beta)$, and there are natural morphisms

$$
\prod_{v \in V\left(\Gamma^{\prime}\right)} \overline{\mathcal{M}}(v) \rightarrow \prod_{v \in V(\Gamma)} \overline{\mathcal{M}}(v),
$$

and the induced one on the fibered products. We denote both of them by $\tilde{\pi}$.

Consider the following commutative diagram

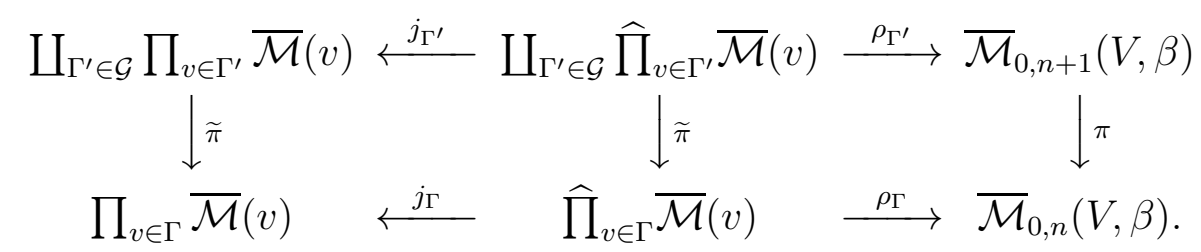

Since $j_{\Gamma *}$ is injective it suffices to show that $j_{\Gamma *} \rho_{\Gamma}^{*} \kappa=\Delta \sum_{v} \kappa(v)$, where $\Delta$ is the Thom class of $\prod_{v} \overline{\mathcal{M}}(v)$ in $\prod_{v} \overline{\mathcal{M}}(v)$.

It suffices to show that

$$
\tilde{\pi}_{*} j_{\Gamma^{\prime} *} \rho_{\Gamma^{\prime}}^{*}=j_{\Gamma *} \rho_{\Gamma}^{*} \pi_{*}
$$

Indeed,

$$
\begin{aligned}
& j_{\Gamma *} \rho_{\Gamma}^{*} \kappa=j_{\Gamma *} \rho_{\Gamma}^{*} \pi_{*}\left(\psi_{n+1}^{a+1} e v_{n+1}\left(e_{\alpha}\right)\right)=\widetilde{\pi}_{*} j_{\Gamma^{\prime} *} \rho_{\Gamma^{\prime}}^{*}\left(\psi_{n+1}^{a+1} e v_{n+1}\left(e_{\alpha}\right)\right) \\
& \quad=\widetilde{\pi}_{*} j_{\Gamma^{\prime} *} j_{\Gamma^{\prime}}^{*}\left(\psi_{n+1}^{a+1} e v_{n+1}\left(e_{\alpha}\right)\right)=\widetilde{\pi}_{*}\left(\widetilde{\Delta} \psi_{n+1}^{a+1} e v_{n+1}\left(e_{\alpha}\right)\right)=\Delta \sum_{v} \kappa(v) .
\end{aligned}
$$


Here we have used (11) for the second equality, Lem. 3.3 for the third equality. We denoted by $\widetilde{\Delta}$ the Thom class of $\coprod_{\Gamma^{\prime} \in \mathcal{G}} \prod_{v \in \Gamma^{\prime}} \overline{\mathcal{M}}(v)$ in $\coprod_{\Gamma^{\prime} \in \mathcal{G}} \prod_{v \in \Gamma^{\prime}} \overline{\mathcal{M}}(v)$, and have used that it is the pull back of $\Delta$ under the map $\widetilde{\pi}$.

In order to show (11) it suffices to prove that $\tilde{\pi}_{*} \rho_{\Gamma^{\prime}}^{*}=\rho_{\Gamma}^{*} \pi_{*}$. This follows from the fact that the right square is the normalization of a pull back square and [11, 6.2].

Our next goal is to express the $\psi$ classes via the boundary strata. Since the action of the symmetric group on the moduli spaces of stable maps interchanges the $\psi$ classes it is enough to give a presentation of $\psi_{1}$ via the boundary strata. We will denote $\psi_{1}$ on $\overline{\mathcal{M}}_{0, n}(V, \beta)$ by $\psi_{(n, \beta)}$. We also assume that if a graph is not stable, then it represents the zero cohomology class.

Lemma 3.5. If $n \geq 3, a \geq 1$, then the following holds in $H^{\bullet}\left(\overline{\mathcal{M}}_{0, n}(V, \beta)\right)$

$$
\psi_{(n, \beta)}^{a}=\sum_{\substack{\beta_{1}+\beta_{2}=\beta \\ I \sqcup J=[n-2] \\ 1 \in J}}^{n-1}
$$

Proof. Getzler [13] and Pandharipande [33] informed us that each of them also has a proof of this lemma (unpublished). It is also stated in 27 that the proof of the lemma for all projective manifolds $V$ in case $a=1$ will appear in [31.

It is enough to prove the lemma for $a=1$. This and Lem. 3.3 will imply the lemma for all $a \geq 1$.

First we prove that

$$
\psi_{(3, \beta)}=\sum_{\beta_{1}+\beta_{2}=\beta}\left[D_{\beta_{1}, \beta_{2}}\right]
$$

where

$$
\left[D_{\beta_{1}, \beta_{2}}\right]=\sum_{\substack{\beta_{1}+\beta_{2}=\beta \\ I \sqcup J=[n-2] \\ 1 \in J}}^{2} \dot{\beta}_{1} \quad \dot{\beta}_{2}
$$

and $D_{\beta_{1}, \beta_{2}}$ is the corresponding divisor. The stability condition implies that $\beta_{2} \neq 0$.

First note that the restriction of $\psi_{(3, \beta)}$ to $U:=\overline{\mathcal{M}}_{0,3}(V, \beta)-\bigcup D_{\beta_{1}, \beta_{2}}$ is zero. Indeed, consider the universal map $\overline{\mathcal{M}}_{0,4}(V, \beta) \rightarrow \overline{\mathcal{M}}_{0,3}(V, \beta)$ restricted to $U$. Let $x \in U$, and $C_{x}$ be the inverse image of $x$ under the universal map. The image of the section of the universal map 
determined by the first marked point lies on the irreducible component of $C_{x}$ which remains stable after forgetting the map $f_{x}: C_{x} \rightarrow V$. This shows that $\psi_{(3, \beta)}$ restricted to $U$ is the pull-back from $\overline{\mathcal{M}}_{0,3}$, and therefore trivial.

It follows from the previous paragraph that

$$
\psi_{(3, \beta)}=\sum_{\beta_{1}+\beta_{2}=\beta, i} k_{\beta_{1}, \beta_{2}, i}\left[D_{\beta_{1}, \beta_{2}, i}\right] \text {, }
$$

where $D_{\beta_{1}, \beta_{2}, i}$ are (disjoint) irreducible components of $D_{\beta_{1}, \beta_{2}}$. In order to determine the coefficients it suffices to intersect (2) with an arbitrary complete non-singular curve $S$ in $\overline{\mathcal{M}}_{0,3}(V, \beta)$. Let $T \rightarrow S$ be a family of 3-pointed stable maps over $S$, and $\nu$ be the corresponding morphism $S \rightarrow \overline{\mathcal{M}}_{0,3}(V, \beta)$. We can assume that the image of $\nu$ intersects each codimension one boundary stratum transversally, and it is disjoint from the intersection of the boundary strata. Then $[S] \cdot \sum\left[D_{\beta_{1}, \beta_{2}}\right]=\sum_{\beta_{1}, \beta_{2}, i} l_{\beta_{1}, \beta_{2}, i}$, where $l_{\beta_{1}, \beta_{2}, i}$ is the number of points in the intersection of $S$ with $D_{\beta_{1}, \beta_{2}, i}$. The stabilization morphism, i.e., the morphism determined by forgetting the map to $V$, determines a morphism $T \rightarrow S \times \mathbb{C P}^{1}$. It is the blow up at the points corresponding to contracted rational components. The image of the first section contains exactly those points which lie in the fibers over $D_{\beta_{1}, \beta_{2}, i} \cap S$. It follows that $\psi_{(3, \beta)} \cdot[S]=\sum\left[D_{\beta_{1}, \beta_{2}}\right] \cdot[S]$, and therefore $\psi_{(3, \beta)}=\sum\left[D_{\beta_{1}, \beta_{2}}\right]$.

In order to obtain the statement of the lemma for $n \geq 4$ one can first show that if $\pi: \overline{\mathcal{M}}_{0, n+1}(V, \beta) \rightarrow \overline{\mathcal{M}}_{0, n}(V, \beta)$ is the universal map, then $\psi_{(n+1, \beta)}=\pi^{*} \psi_{(n, \beta)}+\left[D_{1, n+1}\right]$, where $D_{1, n+1}$ is the image of the first section of the universal map. This can be done by using an argument similar to the above. (See also [23].) Then one need to pull back cohomology classes represented by the boundary strata using the description from Sec. 2 .

As a corollary using Lem. 2.1 we express the $\kappa$ classes via the boundary strata and the $\kappa_{-1}$ classes. We denote the class $\kappa_{a, \alpha}$ on $\overline{\mathcal{M}}_{0, n}(V, \beta)$ by $\kappa_{a, \alpha,(n, \beta)}$.

Corollary 3.6. If $n \geq 2, a \geq 1$, then the following holds in $H^{\bullet}\left(\overline{\mathcal{M}}_{0, n}(V, \beta)\right)$

$$
\kappa_{a, \alpha,(n, \beta)}=\sum_{\substack{\beta_{1}+\beta_{2}=\beta \\ I \sqcup J=[n-2]}}^{n-1} \overbrace{\beta_{1}}^{n} \kappa_{a-1, \alpha}
$$

If $n \geq 2$, then the following holds in $H^{\bullet}\left(\overline{\mathcal{M}}_{0, n}(V, \beta)\right)$ 


$$
\begin{aligned}
& \kappa_{0, \alpha,(n, \beta)}=\sum_{\substack{\beta_{1}+\beta_{2}=\beta \\
I \sqcup J=[n-2]}}^{n-1} \sum_{\beta_{1}}^{n} \kappa_{-1, \alpha} \\
& +\sum_{i \in[n-2]}[n-2]-i \int_{\beta}^{n-1} e^{n} \mathrm{ev}_{i}^{*} e_{\alpha}
\end{aligned}
$$

It follows from the above presentation of the $\kappa$ classes that $\kappa_{0,0}=n-$ $2 \in H^{0}\left(\overline{\mathcal{M}}_{0, n}(V, \beta)\right)$. This can be also seen directly by integrating over the fibers of the universal curve. One can also check that if $\gamma \in H^{2}(V)$, then $\kappa_{-1}(\gamma)=\int_{\beta} \gamma$ on $\overline{\mathcal{M}}_{0, n}(V, \beta)$. We will need this in Sec. 5 .

\section{Cohomological Field Theories}

In this section, we define the notion of a cohomological field theory and prove that the Gromov-Witten invariants twisted by the $\kappa$ classes endows $H^{\bullet}(V)$ with the a family of genus zero CohFT structures. This is equivalent to endowing $H^{\bullet}(V)$ with a family of Frobenius manifold structures (with nonflat identity element, in general) arising from the Poincaré pairing and deformations of the cup product on $V$. Our deformations contain quantum cohomology as a special case.

The moduli spaces of curves $\overline{\mathcal{M}}:=\left\{\overline{\mathcal{M}}_{g, n}\right\}$ forms a modular operad, a higher genus generalization of an operad, [14, 15] in the category of smooth stacks. The action of the symmetric group relabels the marked points while the inclusion of strata gives rise to the composition maps. This endows the homology groups $H_{\bullet}(\overline{\mathcal{M}}):=\left\{H_{\bullet}\left(\overline{\mathcal{M}}_{g, n}\right)\right\}$ with the structure of a modular operad in the category of graded vector spaces. Let $(\mathcal{H}, \eta)$ be a $\left(\mathbb{Z}_{2}\right.$-graded $)$ vector space $\mathcal{H}$ with an even, symmetric, nondegenerate, bilinear form $\eta$, the endomorphism operad is given by $\mathcal{E} n d(\mathcal{H}):=\left\{\mathcal{E} n d_{g, n}(\mathcal{H})\right\}$ where $\mathcal{E} n d_{g, n}(\mathcal{H}):=T^{n} \mathcal{H}^{*}$ for stable pairs $(g, n)$.

Definition 4.1. A (complete) cohomological field theory $(\mathcal{H}, \eta)$ (or CohFT) is a morphism of modular operads $H_{\bullet}(\overline{\mathcal{M}}) \rightarrow \mathcal{E} n d(\mathcal{H})$, i.e. $(\mathcal{H}, \eta)$ is an $H_{\bullet}(\overline{\mathcal{M}})$-algebra. A cohomological field theory of genus $g$ is a sequence of linear maps $H_{\bullet}\left(\overline{\mathcal{M}}_{g^{\prime}, n}\right) \rightarrow \mathcal{E} n d_{g^{\prime}, n}(\mathcal{H})$ for all $g^{\prime} \leq g$ satisfying the subset of axioms of a cohomological field theory which includes only objects of genus $g^{\prime} \leq g$.

There is a dual description of a CohFT in terms of cohomology classes. A CohFT is a pair $(\mathcal{H}, \eta)$ together with a collection $\Omega:=$ $\left\{\Omega_{g, n}\right\}$ where $\Omega_{g, n}$ is an even element in $\mathcal{R}_{g, n}:=H^{\bullet}\left(\overline{\mathcal{M}}_{g, n}\right) \otimes T^{n} \mathcal{H}^{*}$ 
defined for stable pairs $(g, n)$ satisfying the following (where the summation convention has been used):

i: $\Omega_{g, n}$ is invariant under the action of the symmetric group $S_{n}$.

ii: For each partition of $[n]=S_{1} \sqcup S_{2}$ such that $\left|S_{1}\right|=n_{1}$ and $\left|S_{2}\right|=n_{2}$ and nonnegative $g_{1}, g_{2}$ such that $g=g_{1}+g_{2}$ and $2 g_{i}-2+n_{i}>0$ for all $i$, consider the inclusion map $\rho: \overline{\mathcal{M}}_{g_{1}, S_{1} \sqcup *} \times$

$\overline{\mathcal{M}}_{g_{2}, S_{2} \sqcup *} \rightarrow \overline{\mathcal{M}}_{g_{1}+g_{2}, n}$ where $*$ denotes the two marked points that are attached under the inclusion map. The forms satisfy the restriction property

$$
\begin{aligned}
& \rho^{*} \Omega_{g, n}\left(\gamma_{1}, \gamma_{2} \ldots, \gamma_{n}\right)= \\
& \pm \Omega_{g_{1}, n_{1}}\left(\left(\bigotimes_{\alpha \in S_{1}} \gamma_{\alpha}\right) \otimes e_{\mu}\right) \eta^{\mu \nu} \otimes \Omega_{g_{2}, n_{2}}\left(e_{\nu} \otimes \bigotimes_{\alpha \in S_{2}} \gamma_{\alpha}\right)
\end{aligned}
$$

where the sign \pm is the usual one obtained by applying the permutation induced by $S$ to $\left(\gamma_{1}, \gamma_{2}, \ldots \gamma_{n}\right)$ taking into account the grading of $\left\{\gamma_{i}\right\}$ and where $\left\{e_{\alpha}\right\}$ is a homogeneous basis for $\mathcal{H}$.

iii: Let $\chi: \overline{\mathcal{M}}_{g-1, n+2} \rightarrow \overline{\mathcal{M}}_{g, n}$ be the canonical map corresponding to attaching the last two marked points together then

$$
\chi^{*} \Omega_{g, n}\left(\gamma_{1}, \gamma_{2}, \ldots, \gamma_{n}\right)=\Omega_{g-1, n+2}\left(\gamma_{1}, \gamma_{2}, \ldots, \gamma_{n}, e_{\mu}, e_{\nu}\right) \eta^{\mu \nu} .
$$

Let $\Gamma$ be a stable graph then there is a canonical map $\rho_{\Gamma}$ obtained by composition of the canonical maps

$$
\prod_{v \in V(\Gamma)} \overline{\mathcal{M}}_{g(v), n(v)} \rightarrow \overline{\mathcal{M}}_{\Gamma} \rightarrow \overline{\mathcal{M}}_{g, n}
$$

Since the map $\rho_{\Gamma}$ can be constructed from morphisms in (ii) and (iii) above, $\Omega_{g, n}$ satisfies a restriction property of the form

$$
\rho_{\Gamma}^{*} \Omega_{g, n}=P_{\Gamma}\left(\bigotimes_{v \in V(\Gamma)} \Omega_{g(v), n(v)}\right)
$$

where

$$
P_{\Gamma}: \bigotimes_{v \in V(\Gamma)} \mathcal{R}_{g(v), n(v)} \rightarrow \mathcal{R}_{g, n}
$$

is the linear map contracting tensor factors of $\mathcal{H}$ using the metric $\eta$ induced from successive application of equations (ii) and (iii) above.

The collection $\Omega$ forms a (complete) $\operatorname{CohFT} H_{\bullet}\left(\overline{\mathcal{M}}_{g, n}\right) \rightarrow \mathcal{E} n d_{g, n}(\mathcal{H})$ via $[c] \mapsto \int_{[c]} \Omega_{g, n}$. It is this formulation of CohFTs which arises most naturally in algebraic geometry.

Notice that the definition of a cohomological field theory is valid even when enlarges the ground ring from $\mathbb{C}$ to another ring $\mathcal{K}$. 
From now on, we shall restrict our consideration to genus zero CohFTs.

Notation . Let $V$ be a topological space and let $H^{\bullet}(V, \mathbb{C})$ be given a homogeneous basis $\mathbf{e}:=\left\{e_{\alpha}\right\}_{\alpha \in A}$ and let $e_{0}$ denote the identity element. Let $\mathbf{s}:=\left\{s_{r}^{\alpha} \mid r \geq-1, \alpha \in A\right\}$ be a collection of formal variables with grading $\left|s_{r}^{\alpha}\right|=2 r+\left|e_{\alpha}\right|$. All formal power series and polynomials in a collection of variables (e.g. s) are in the $\mathbb{Z}_{2}$-graded sense.

Definition 4.2. Let $\Lambda$ consist of formal symbols $q^{\beta}$ for all $\beta \in B(V)$ together with the multiplication $\left(q^{\beta} q^{\beta^{\prime}}\right) \mapsto q^{\beta+\beta^{\prime}}$. Let $\mathbb{C}[[\Lambda]]$ consist of formal sums $\sum_{\beta \in B(V)} a_{\beta} q^{\beta}$ where $a_{\beta}$ are elements in $\mathbb{C}$. Assign to each $q^{\beta}$, the degree $-2 c_{1}(V) \cap \beta$. The product is well-defined according to [24, Prop. II.4.8]. This endows $\Lambda$ with the structure of a semigroup with unit. Furthermore, let $\mathbb{C}[[\Lambda, \mathbf{s}]]:=\mathbb{C}[[\Lambda]][[\mathbf{s}]]$, formal power series in the variables $\mathbf{s}$ with coefficients in $\mathbb{C}[[\Lambda]]$.

Suppose that $H_{2}(V, \mathbb{Z})$ has no torsion then the semigroup $\Lambda$ can be realized concretely by choosing a basis $\left\{e^{\epsilon}\right\}$ for $H_{2}(V, \mathbb{Z}$ ) (say the one which is a subset of the basis dual via the Poincaré pairing to $\left.\left\{e_{\alpha}\right\}\right)$, introducing a formal variable $q_{\epsilon}$ for each such basis element, writing $\beta=\sum_{\epsilon} \beta_{\epsilon} e^{\epsilon}$, and defining $q^{\beta}=\prod_{\epsilon} q_{\epsilon}^{\beta_{\epsilon}}$. The variable $q_{\epsilon}$ is then assigned the degree $-2 c_{1}(V) \cap e^{\epsilon}$. If $H_{2}(V, \mathbb{Z})$ contains a torsion subgroup decomposed into a product of cyclic groups then one can do the same by introducing a "root of unity" for each generator of a cyclic subgroup in the obvious manner.

Theorem 4.3. Let $V$ be a smooth, projective, convex variety. For each $n \geq 3$, let $\Omega_{0, n}$ be elements in $\mathcal{R}_{0, n}(V)[[\Lambda, \mathbf{s}]]$ defined by

$$
\Omega_{0, n}\left(\gamma_{1}, \ldots, \gamma_{n}\right):=\sum_{\beta} \mathrm{st}_{*}\left(e v_{1}^{*} \gamma_{1} \cdots e v_{n}^{*} \gamma_{n} \exp (\mathbf{s} \boldsymbol{\kappa})\right) q^{\beta}
$$

where st $: \overline{\mathcal{M}}_{0, n}(V, \beta) \rightarrow \overline{\mathcal{M}}_{0, n}$ is the forgetful map, $\gamma_{1}, \gamma_{2}, \ldots, \gamma_{n}$ are elements in $H^{\bullet}(V, \mathbb{C})$. The $\Omega:=\left\{\Omega_{0, n}\right\}$ endows $\left(H^{\bullet}(V, \mathbb{C}[[\Lambda, \mathbf{s}]]), \eta\right)$ with the structure of a CohFT where $\eta$ is the Poincaré pairing extended $\mathbb{C}[[\Lambda, \mathbf{s}]]$-linearly. In particular, if $H^{\bullet}(V, \mathbb{C})$ consists of only even dimensional cohomology classes then for all values of $\left\{s_{r}^{\alpha}\right\}, \Omega:=\left\{\Omega_{0, n}\right\}$ endows $\left(H^{\bullet}(V, \mathbb{C}[[\Lambda]]), \eta\right)$ with the structure of a CohFT where $\eta$ is the Poincaré pairing.

Proof. Let $n \geq 3$, and let $\Gamma$ be a stable graph for $\overline{\mathcal{M}}_{0, n}$. Let $\mathcal{G}$ be the set of graphs $\Gamma^{\prime}$ obtained by decorating the vertices of $\Gamma$ with elements $\beta(v) \in H_{2}(V)$ such that $\sum_{v \in V(\Gamma)} \beta(v)=\beta$. Clearly, each $\Gamma^{\prime} \in \mathcal{G}$ 
determines a stratum in $\overline{\mathcal{M}}_{0, n}(V, \beta)$. It follows from the deformation theory considerations in [12] that the union of $\overline{\mathcal{M}}_{\Gamma^{\prime}}, \Gamma^{\prime} \in \mathcal{G}$, is the scheme theoretic preimage of $\overline{\mathcal{M}}_{\Gamma}$ under the morphism st.

Consider the following commuting diagram:

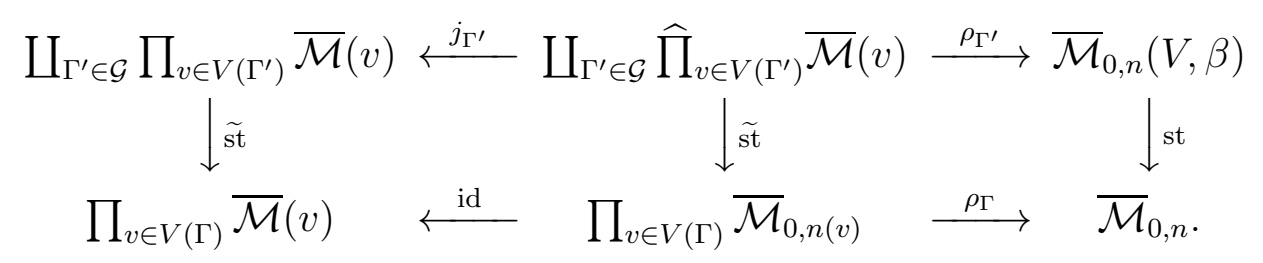

One has to check that the element $\Omega_{0, n}\left(\gamma_{1}, \ldots, \gamma_{n}\right)$ defined by (田) possesses the restriction property (3). It suffices to check it for each $\beta$. Since the right square of the commutative diagram is the normalization of a pull-back square one has that $\rho_{\Gamma}^{*} \mathrm{st}_{*}=\sum_{\Gamma^{\prime} \in \mathcal{G}} \widetilde{\mathrm{st}}_{*} j_{\Gamma^{\prime} *} \rho_{\Gamma^{\prime}}^{*}$ [11, 6.2]. The required restriction property follows from the following facts. Firstly, for each class $\kappa=\kappa_{a, \alpha}$ one has $\rho_{\Gamma^{\prime}}^{*} \kappa=\sum_{v \in V\left(\Gamma^{\prime}\right)} j_{\Gamma^{\prime}}^{*} \kappa(v)$, where $\kappa(v)$ is the corresponding class on $\overline{\mathcal{M}}(v)$, as shown in Lem. 3.4. Secondly, $\rho_{\Gamma^{\prime}}^{*} e v_{i}^{*} \gamma_{i}=j_{\Gamma^{\prime}}^{*} e v_{(v), i}^{*}\left(\gamma_{i}\right)$, where $v$ is the vertex of $\Gamma^{\prime}$ to which the tail labeled $i$ attached, and $e v_{(v), i}$ is the corresponding evaluation morphism. Thirdly, the composition $j_{\Gamma^{\prime} *} j_{\Gamma^{\prime}}^{*}$ is the multiplication of by the Thom class of the fibered product in the corresponding direct product.

Finally, suppose that $H^{\bullet}(V)$ consists of only even dimensional classes. By dimensional considerations and the fact that $\kappa_{0,0}=n-2$ and $\kappa_{-1}(\gamma)=\int_{\beta} \gamma$ when $\gamma$ has degree 2 , the coefficient of $q^{\beta}$ in $\Omega_{0, n}$ converges when one plugs in numbers for $s_{n}^{\alpha}$ for all $n=-1,0, \ldots$ and $\alpha=0,1, \ldots, r$.

There is a third way of describing CohFTs and this is in terms of a generating function called its potential. We will see in the next section that when considering generating functions for the intersection numbers involving combinations of $\psi$ classes as well, this alternate description of CohFTs will be more useful.

Definition 4.4. The potential $\Phi$ of a genus zero CohFT is given by a linear map $\mu: H_{\bullet}\left(\overline{\mathcal{M}}_{0, \bullet}, \mathbb{C}\right) \rightarrow \mathcal{E} n d(\mathcal{H})$ of rank $r+1$ is defined by choosing a basis $\left\{e_{0}, \ldots, e_{r}\right\}$ for $\mathcal{H}$ and let $\mathbf{x}=\sum_{\alpha=0}^{r} x^{\alpha} e_{\alpha}$ represent an arbitrary cohomology class in $\mathcal{H}$. Let $I_{0, n}(\mathbf{x}, \mathbf{x}, \cdots, \mathbf{x})$ be the element in $\mathbb{C}\left[\left[x^{0}, x^{1} \ldots, x^{r}\right]\right]$, the ring of formal power series with $\mathbb{C}$ coefficients in $x^{0}, \ldots, x^{r}$ in the graded sense, obtained by using $h$ to 
pair $\mu_{0, n}\left(\left[\overline{\mathcal{M}}_{0, n}\right]\right)$ with $\mathbf{x} \otimes \mathbf{x} \otimes \ldots \otimes \mathbf{x}$ and

$$
\Phi(\mathbf{x}):=\sum_{n=3}^{\infty} I_{0, n}(\mathbf{x}, \mathbf{x}, \ldots, \mathbf{x}) \frac{1}{n !}
$$

which is regarded as an element in $\mathbb{C}\left[\left[x^{0}, \ldots, x^{r}\right]\right]$.

This definition generalizes to arbitrary genera in the obvious manner. The potential completely characterizes the cohomological field theory in genus zero as can be seen from the following theorem.

Theorem 4.5. An element $\Phi$ in $\mathbb{C}\left[\left[x^{0}, \ldots, x^{r}\right]\right]$ is the potential of a rank $r$, genus zero $\operatorname{CohFT}(\mathcal{H}, \eta)$ if and only if [26, 30] it contains only terms which are cubic and higher order in the variables $x^{0}, \ldots, x^{r}$ and it satisfies the WDVV equation

$$
\left(\partial_{a} \partial_{b} \partial_{e} \Phi\right) \eta^{e f}\left(\partial_{f} \partial_{c} \partial_{d} \Phi\right)=(-1)^{\left|x_{a}\right|\left(\left|x_{b}\right|+\left|x_{c}\right|\right)}\left(\partial_{b} \partial_{c} \partial_{e} \Phi\right) \eta^{e f}\left(\partial_{f} \partial_{a} \partial_{d} \Phi\right)
$$

where $\eta_{a b}:=\eta\left(e_{a}, e_{b}\right), \eta^{a b}$ is in inverse matrix to $\eta_{a b}, \partial_{a}$ is derivative with respect to $x^{a}$, and the summation convention has been used.

The theorem is a consequence of the work of Keel [22] who proved that all relations between boundary divisors in $H_{\bullet}\left(\overline{\mathcal{M}}_{0, n}\right)$ arise from lifting the basic codimension one relation on $\overline{\mathcal{M}}_{0, n}$.

As before, one can extend the ground ring $\mathbb{C}$ above to $\mathbb{C}[[\Lambda, \mathbf{s}]]$ in the definition of the potential of a genus zero CohFT and the above theorem extends, as well. In our setting, the potential is a formal function on $\mathcal{H}:=H^{\bullet}(V, \mathbb{C}[[\Lambda, \mathbf{s}]])$ of the following kind. $\Phi$ belongs to $\mathbb{C}[[\Lambda, \mathbf{s}]]\left[\left[x^{0}, \ldots, x^{r}\right]\right]$ where we have used

$$
I_{0, n}\left(e_{\alpha_{1}}, \ldots, e_{\alpha_{n}}\right)=\int_{\overline{\mathcal{M}}_{0, n}}\left\langle\Omega_{0, n}, e_{\alpha_{1}} \otimes e_{\alpha_{2}} \otimes \cdots \otimes e_{\alpha_{n}}\right\rangle .
$$

Again, if $H^{\bullet}(V)$ consists entirely of even dimensional classes then plugging in numbers for all $s_{n}^{\alpha}$ where $n=-1,0,1, \ldots$ and $\alpha=0,1, \ldots, r$, one obtains families of CohFT structures on $H^{\bullet}(V, \mathbb{C}[[\Lambda]])$.

\section{Topological Recursion Relations}

In this section we will derive differential equations for the generating function which incorporates the intersection numbers of the $\psi$ and $\kappa$ classes.

Notation . Let $\mathcal{S}_{k}^{r}$ be the set of infinite sequences of non-negative integers

$$
\mathbf{m}=\left(m_{k}^{0}, m_{k}^{1}, \ldots, m_{k}^{r}, m_{k+1}^{0}, \ldots, m_{k+1}^{r}, m_{k+2}^{0}, \ldots\right)
$$


such that $m_{a}^{\alpha}=0$ for all a sufficiently large. We denote by $\boldsymbol{\delta}_{a}^{\alpha}$ the infinite sequence whose only non-zero entry is $m_{a}^{\alpha}=1$. We use the Latin characters for the lower index and the Greek characters for the upper index. We will use the notation of the type

$$
\mathbf{m} !:=\prod_{\alpha=0}^{r} \prod_{a \geq k} m_{a}^{\alpha} ! \quad \text { and } \quad\left(\begin{array}{c}
\mathbf{m} \\
\mathbf{m}^{\prime}
\end{array}\right):=\frac{\mathbf{m} !}{\mathbf{m}^{\prime} !\left(\mathbf{m}-\mathbf{m}^{\prime}\right) !}
$$

We also consider $\mathbf{t}=\left\{t_{d}^{\mu}\right\}, d \geq 0, \mu=0, \ldots, r, \mathbf{s}=\left\{s_{a}^{\alpha}\right\}, a \geq$ $-1, \alpha=0, \ldots, r$, be a collection of independent formal variables with grading $\left|t_{a}^{\alpha}\right|=2 a-2+\left|e_{\alpha}\right|$ and $\left|s_{a}^{\alpha}\right|=2 a+\left|e_{\alpha}\right|$.

5.1. Differential Equations. We start with introducing the notation for the intersection numbers. This notation generalizes the one from 37, 18.

Definition 5.1. Let $\beta \in H_{2}(V, \mathbb{Z})$, and $\left\{e_{\alpha}\right\}, \alpha=0, \ldots, r$ is a basis of $H^{\bullet}(V)$. If $\beta \neq 0$, or $\beta=0$ and $n \geq 3$, then

$$
\begin{gathered}
\left\langle\tau_{d_{1}}^{\mu_{1}} \tau_{d_{2}}^{\mu_{2}} \ldots \tau_{d_{n}}^{\mu_{n}} \kappa_{a_{1}, \alpha_{1}}, \kappa_{a_{2}, \alpha_{2}} \ldots \kappa_{a_{l}, \alpha_{l}}\right\rangle_{\beta}:= \\
\int_{\overline{\mathcal{M}}_{0, n}(V, \beta)} t_{d_{1}}^{\mu_{1}} \psi_{1}^{d_{1}} e v_{1}^{*}\left(e_{\mu_{1}}\right) t_{d_{2}}^{\mu_{2}} \psi_{2}^{d_{2}} e v_{2}^{*}\left(e_{\mu_{2}}\right) \ldots t_{d_{n}}^{\mu_{n}} \psi_{n}^{d_{n}} e v_{n}^{*}\left(e_{\mu_{n}}\right) \times \\
s_{a_{1}}^{\alpha_{1}} \kappa_{a_{1}, \alpha_{1}} s_{a_{2}}^{\alpha_{2}} \kappa_{a_{2}, \alpha_{2}} \ldots s_{a_{l}}^{\alpha_{l}} \kappa_{a_{l}, \alpha_{l}}=(-1)^{\epsilon} t_{d_{1}}^{\mu_{1}} \ldots t_{d_{n}}^{\mu_{n}} s_{a_{1}}^{\alpha_{1}} \ldots s_{a_{l}}^{\alpha_{l}} \times \\
\int_{\overline{\mathcal{M}}_{0, n}(V, \beta)} \psi_{1}^{d_{1}} e v_{1}^{*}\left(e_{\mu_{1}}\right) \ldots \psi_{n}^{d_{n}} e v_{n}^{*}\left(e_{\mu_{n}}\right) \kappa_{a_{1}, \alpha_{1}} \ldots \kappa_{a_{l}, \alpha_{l}} .
\end{gathered}
$$

If $\beta=0$ and $n<3$ we define all intersection numbers to be equal to 0 .

Let the sequence $\left(d_{1}, \mu_{1}\right),\left(d_{2}, \mu_{2}\right), \ldots,\left(d_{n}, \mu_{n}\right)$ contain $m_{d}^{\mu}$ pairs $(d, \mu)$, where $d \geq 0,0 \leq \mu \leq r$, and the sequence $\left(a_{1}, \alpha_{1}\right),\left(a_{2}, \alpha_{2}\right), \ldots,\left(a_{l}, \alpha_{l}\right)$ contain $p_{a}^{\alpha}$ pairs $(a, \alpha)$ where $a \geq-1,0 \leq \alpha \leq r$. Then we also denote the intersection number above by $\left\langle\boldsymbol{\tau}^{\mathbf{m}} \boldsymbol{\kappa}^{\mathbf{p}}\right\rangle_{\beta}$.

We also set

$$
\left\langle\boldsymbol{\tau}^{\mathbf{m}} \boldsymbol{\kappa}^{\mathbf{p}}\right\rangle:=\sum_{\beta}\left\langle\boldsymbol{\tau}^{\mathbf{m}} \boldsymbol{\kappa}^{\mathbf{p}}\right\rangle_{\beta} q^{\beta}
$$

where $q$ is the formal variable introduced in the previous section.

Remark. We put the formal variables in the definition of the intersection numbers in order to take care of the signs provided $V$ has odd cohomology classes. If $V$ has only even cohomology classes, then $\epsilon$ in the formula above is 0 . Then setting all formal $t$ variables equal to 1 and all formal $s$ variables equal to 0 we obtain the definition due to Witten [37. In this paper we assume that $V$ may have odd cohomology classes even though we do not know examples of convex manifolds with odd cohomology classes. 
Definition 5.2. We define $H(\mathbf{t} ; \mathbf{s})$ in $\mathbb{C}[[\Lambda, \mathbf{t}, \mathbf{s}]]$ by

$$
H(\mathbf{t} ; \mathbf{s}):=\sum_{\mathbf{m} \in \mathcal{S}_{0}^{r} \mathbf{p} \in \mathcal{S}_{-1}^{r}} \frac{1}{\mathbf{m} !} \frac{1}{\mathbf{p} !}\left\langle\boldsymbol{\tau}^{\mathbf{m}} \boldsymbol{\kappa}^{\mathbf{p}}\right\rangle
$$

Notice that $H$ has grading $2\left(\operatorname{dim}_{\mathbb{C}} V-3\right)$.

We are going to exploit the presentations of the $\psi$ classes and the $\kappa$ classes in Lem. 3.5 and Cor. 3.6 in order to obtain a system of differential equations for $H$. The explicit presentation of the tautological classes allows one to obtain recursion relations for the intersection numbers, and these recursion relations in turn provide the corresponding differential equations. The technical details are standard (cf. [37, 21, 18]), and we omit them.

We recall that we denote by $\eta$ the Poincaré pairing on $H^{\bullet}(V)$. We denote $\eta\left(e_{\alpha_{1}}, e_{\alpha_{2}}\right)$ by $\eta_{\alpha_{1}, \alpha_{2}}$, and by $\eta^{\alpha_{1}, \alpha_{2}}$ the coefficients of the inverse metric. We denote by $c_{\alpha_{2}, \alpha_{3}}^{\alpha_{1}}$ the coefficients of the multiplication tensor, that is

$$
e_{\alpha_{2}} e_{\alpha_{3}}=\sum_{\alpha_{1}=0}^{r} c_{\alpha_{2}, \alpha_{3}}^{\alpha_{1}} e_{\alpha_{1}}
$$

Proposition 5.3. The intersection numbers defined above satisfy the recursion relations below called the topological recursion relations. The first two relations are valid when $a_{1} \geq 1$.

$$
\begin{aligned}
& \left(t_{a_{1}}^{\alpha_{1}}\right)^{-1}\left\langle\boldsymbol{\tau}^{\mathbf{m}+\boldsymbol{\delta}_{a_{1}}^{\alpha_{1}}+\boldsymbol{\delta}_{a_{2}}^{\alpha_{2}}+\boldsymbol{\delta}_{a_{3}}^{\alpha_{3}}} \boldsymbol{\kappa}^{\mathbf{p}}\right\rangle_{\beta}=\left(t_{a_{1}-1}^{\alpha_{1}}\right)^{-1}\left(t_{0}^{\sigma_{1}}\right)^{-1}\left(t_{0}^{\sigma_{2}}\right)^{-1} \times \\
& \sum\left(\begin{array}{c}
\mathbf{m} \\
\mathbf{m}^{\prime}
\end{array}\right)\left(\begin{array}{c}
\mathbf{p} \\
\mathbf{p}^{\prime}
\end{array}\right)\left\langle\boldsymbol{\tau}^{\mathbf{m}^{\prime}+\boldsymbol{\delta}_{a_{1}-1}^{\alpha_{1}}+\boldsymbol{\delta}_{0}^{\sigma_{1}}} \boldsymbol{\kappa}^{\mathbf{p}^{\prime}}\right\rangle_{\beta_{1}} \eta^{\sigma_{1}, \sigma_{2}}\left\langle\boldsymbol{\tau}^{\mathbf{m}^{\prime \prime}+\boldsymbol{\delta}_{0}^{\sigma_{2}}+\boldsymbol{\delta}_{a_{2}}^{\alpha_{2}}+\boldsymbol{\delta}_{a_{3}}^{\alpha_{3}}} \boldsymbol{\kappa}^{\mathbf{p}^{\prime \prime}}\right\rangle_{\beta_{2}}
\end{aligned}
$$

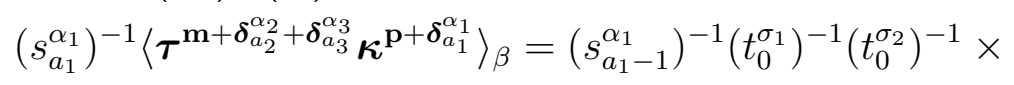

$$
\begin{aligned}
& \sum\left(\begin{array}{c}
\mathbf{m} \\
\mathbf{m}^{\prime}
\end{array}\right)\left(\begin{array}{c}
\mathbf{p} \\
\mathbf{p}^{\prime}
\end{array}\right)\left\langle\boldsymbol{\tau}^{\mathbf{m}^{\prime}+\boldsymbol{\delta}_{0}^{\sigma_{1}}} \boldsymbol{\kappa}^{\mathbf{p}^{\prime}+\boldsymbol{\delta}_{a_{1}-1}^{\alpha_{1}}}\right\rangle_{\beta_{1}} \eta^{\sigma_{1}, \sigma_{2}}\left\langle\boldsymbol{\tau}^{\mathbf{m}^{\prime \prime}+\boldsymbol{\delta}_{0}^{\sigma_{2}}+\boldsymbol{\delta}_{a_{2}}^{\alpha_{2}}+\boldsymbol{\delta}_{a_{3}}^{\alpha_{3}}} \boldsymbol{\kappa}^{\mathbf{p}^{\prime \prime}}\right\rangle_{\beta_{2}} \\
& \left(s_{0}^{\alpha_{1}}\right)^{-1}\left\langle\boldsymbol{\tau}^{\mathbf{m}+\boldsymbol{\delta}_{a_{2}}^{\alpha_{2}}+\boldsymbol{\delta}_{a_{3}}^{\alpha_{3}}} \boldsymbol{\kappa}^{\mathbf{p}+\boldsymbol{\delta}_{0}^{\alpha_{1}}}\right\rangle_{\beta}=\left(s_{-1}^{\alpha_{1}}\right)^{-1}\left(t_{0}^{\sigma_{1}}\right)^{-1}\left(t_{0}^{\sigma_{2}}\right)^{-1} \times \\
& \sum\left(\begin{array}{c}
\mathbf{m} \\
\mathbf{m}^{\prime}
\end{array}\right)\left(\begin{array}{c}
\mathbf{p} \\
\mathbf{p}^{\prime}
\end{array}\right)\left\langle\boldsymbol{\tau}^{\mathbf{m}^{\prime}+\boldsymbol{\delta}_{0}^{\sigma_{1}}} \boldsymbol{\kappa}^{\mathbf{p}^{\prime}+\boldsymbol{\delta}_{-1}^{\alpha_{1}}}\right\rangle_{\beta_{1}} \eta^{\sigma_{1}, \sigma_{2}}\left\langle\boldsymbol{\tau}^{\mathbf{m}^{\prime \prime}+\boldsymbol{\delta}_{0}^{\sigma_{2}}+\boldsymbol{\delta}_{a_{2}}^{\alpha_{2}}+\boldsymbol{\delta}_{a_{3}}^{\alpha_{3}}} \boldsymbol{\kappa}^{\mathbf{p}^{\prime \prime}}\right\rangle_{\beta_{2}}+
\end{aligned}
$$

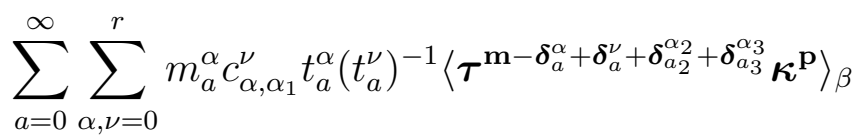

where the summation is over $\beta_{1}+\beta_{2}=\beta, \mathbf{m}^{\prime}+\mathbf{m}^{\prime \prime}=\mathbf{m}, \mathbf{p}^{\prime}+\mathbf{p}^{\prime \prime}=\mathbf{p}$, and $\sigma_{1}, \sigma_{2}$ varying from 0 to $r$. 
Equivalently, the function $H(\mathbf{t} ; \mathbf{s})$ satisfies the system of differential equations below. The first two equations are valid when $a_{1} \geq 1$.

$$
\begin{aligned}
\frac{\partial^{3} H}{\partial t_{a_{1}}^{\alpha_{1}} \partial t_{a_{2}}^{\alpha_{2}} \partial t_{a_{3}}^{\alpha_{3}}} & =\sum_{\sigma_{1}, \sigma_{2}} \frac{\partial^{2} H}{\partial t_{a_{1}-1}^{\alpha_{1}} \partial t_{0}^{\sigma_{1}}} \eta^{\sigma_{1}, \sigma_{2}} \frac{\partial^{3} H}{\partial t_{0}^{\sigma_{2}} \partial t_{a_{2}}^{\alpha_{2}} \partial t_{a_{3}}^{\alpha_{3}}} \\
\frac{\partial^{3} H}{\partial s_{a_{1}}^{\alpha_{1}} \partial t_{a_{2}}^{\alpha_{2}} \partial t_{a_{3}}^{\alpha_{3}}} & =\sum_{\sigma_{1}, \sigma_{2}} \frac{\partial^{2} H}{\partial s_{a_{1}-1}^{\alpha_{1}} \partial t_{0}^{\sigma_{1}}} \eta^{\sigma_{1}, \sigma_{2}} \frac{\partial^{3} H}{\partial t_{0}^{\sigma_{2}} \partial t_{a_{2}}^{\alpha_{2}} \partial t_{a_{3}}^{\alpha_{3}}} \\
\frac{\partial^{3} H}{\partial s_{0}^{\alpha_{1}} \partial t_{a_{2}}^{\alpha_{2}} \partial t_{a_{3}}^{\alpha_{3}}} & =\sum_{\sigma_{1}, \sigma_{2}} \frac{\partial^{2} H}{\partial s_{-1}^{\alpha_{1}} \partial t_{0}^{\sigma_{1}}} \eta^{\sigma_{1}, \sigma_{2}} \frac{\partial^{3} H}{\partial t_{0}^{\sigma_{2}} \partial t_{a_{2}}^{\alpha_{2}} \partial t_{a_{3}}^{\alpha_{3}}} \\
& +\sum_{a=0}^{\infty} \sum_{\alpha, \nu=0}^{r} c_{\alpha, \alpha_{1}}^{\nu} t_{a}^{\alpha} \frac{\partial^{3} H}{\partial t_{a}^{\nu} \partial t_{a_{2}}^{\alpha_{2}} \partial t_{a_{3}}^{\alpha_{3}}},
\end{aligned}
$$

where the indices $\sigma_{1}, \sigma_{2}$ vary from 0 to $r$.

Remark. If one considers the function $F(\mathbf{t}):=H(\mathbf{t} ; \mathbf{0})$, then the first equations reduce to the equations first written by Witten in [37. Witten showed [37] that the WDVV equations in Th. 4.5 follow from the differential equation for $F(\mathbf{t})$.

Corollary 5.4. Let $H$ be as above. Let $x^{\alpha}:=t_{0}^{\alpha}, \mathbf{x}:=\left(x^{0}, \ldots, x^{r}\right)$, and let $\mathbf{t}:=(\mathbf{x}, \widetilde{\mathbf{t}})$ where $\tilde{t}$ consists of all $t_{n}^{\alpha}$ such that $n \geq 1$. Let $\Phi(\mathbf{x}):=H(\mathbf{x}, \widetilde{\mathbf{t}} ; \mathbf{s})$ be regarded as an element of $\mathbb{C}[[\Lambda, \widetilde{\mathbf{t}}, \mathbf{s}]][[\mathbf{x}]]$ then $\Phi$ endows $H^{\bullet}(V, \mathbb{C}[[\Lambda, \widetilde{\mathbf{t}}, \mathbf{s}]])$ with the structure of a CohFT (over the ground ring $\mathbb{C}[[\Lambda, \widetilde{\mathbf{t}}, \mathbf{s}]])$. If $H^{\bullet}(V, \mathbb{C})$ consists of only even dimensional classes then for each value of $\widetilde{\mathbf{t}}$ and $\mathbf{s}, \Phi$ endows $H^{\bullet}(V, \mathbb{C}[[\Lambda]])$ with the structure of a genus zero CohFT (over the ground ring $\mathbb{C}[[\Lambda]]$ ).

Proof. Our proof follows that in [37] but keeping track of signs. Differentiate the first set of equations in the last theorem with respect to the variable $t_{a_{4}}^{\alpha_{4}}$, setting $a_{1}=1$ and $a_{2}=a_{3}=a_{4}=0$ and noting that the left hand side is (graded)symmetric under permutation of the subscripts $2,3,4$. Therefore, the antisymmetric part of the right hand side must vanish. This is nothing more than the WDVV equations (with the correct signs). This implies that $4.5 \Phi$ is a potential for a genus zero CohFT. The fact that the potential $\Phi$ converges for all values of $\widetilde{\mathbf{t}}$ and $\mathbf{s}$ follows from the grading.

5.2. Puncture and Dilaton Equations. Here we introduce analogues of the puncture and the dilaton equations. Using these equations and the topological recursion relations we will show that the intersection numbers defined above can be expressed in terms of GromovWitten invariants. 
For the rest of this subsection let us fix the following notation. Let $\pi: \overline{\mathcal{M}}_{0, n+1}(V, \beta) \rightarrow \overline{\mathcal{M}}_{0, n}(V, \beta)$ denote the universal stable map which "forgets" the $n+1^{\text {st }}$ marked point. To distinguish the $\psi$ and the $\kappa$ classes upstairs and downstairs we put "hats" over the former. Since the pull back of $e v_{i}\left(e_{\alpha}\right)$ is still $e v_{i}\left(e_{\alpha}\right)$ we denote these classes by the same symbol unstairs and downstairs.

In addition, if $\mathbf{m}=\left\{m_{a}^{\alpha}\right\} \in \mathcal{S}_{k}^{r}, k=-1,0$, then

$$
|\mathbf{m}|:=\sum_{\alpha=0}^{r} \sum_{a \geq 0} a m_{a}^{\alpha}, \quad\|\mathbf{m}\|:=\sum_{\alpha=0}^{r} \sum_{a \geq 0} m_{a}^{\alpha}
$$

and if $\mathbf{m}=\left\{m_{a}^{\alpha}\right\} \in \mathcal{S}_{0}^{r}$, then

$$
\begin{aligned}
e(\mathbf{m}) & :=e_{0}^{m_{0}^{0}} \ldots e_{r}^{m_{0}^{r}} e_{0}^{m_{1}^{0}} \ldots e_{r}^{m_{1}^{r}} e_{0}^{m_{2}^{0}} \ldots \\
\mathbf{s}^{\mathbf{m}} & :=\ldots\left(s_{2}^{0}\right)^{m_{2}^{0}}\left(s_{1}^{r}\right)^{m_{1}^{r}} \ldots\left(s_{1}^{0}\right)^{m_{1}^{0}}\left(s_{0}^{r}\right)^{m_{0}^{r}} \ldots\left(s_{0}^{0}\right)^{m_{0}^{0}}
\end{aligned}
$$

Note that in both cases we have summation over $a \geq 0$, not over $a \geq k$, and that the order of the terms in the products is the opposite. We denote by $c_{e(\mathbf{m}), \mu}^{\nu}$ the coefficients of $e_{\nu}$ in the product $e(\mathbf{m}) e_{\mu}$. We also say that $\mathbf{m} \in \mathcal{S}_{0,0}^{r}$ if $\mathbf{m} \in \mathcal{S}_{0}^{r}$, and $m_{a}^{\alpha}=0$ provided $a \geq 1$.

It is easy to derive the following formulas for the pull back of the $\psi$ and $\kappa$ classes with respect to $\pi$ (cf. [33]). Namely,

$$
\widehat{\psi}_{i}^{a}=\pi^{*} \psi_{i}^{a}+\pi^{*} \psi_{i}^{a-1} D_{i} \quad \text { and } \quad \widehat{\kappa}_{a, \alpha}=\pi^{*} \kappa_{a, \alpha}+\widehat{\psi}_{n+1}^{a} e v_{n+1}^{*}\left(e_{\alpha}\right) .
$$

The pull back formulas above induce the recursion relations (5) below. These are analogues of the puncture and dilaton equations. The proof is standard, and we omit the details (cf. [18, Sec. 3.1]). The variables take care of the signs as in the case of the topological recursion relations. When $a \geq 1$, or when $a=0$ and $\mathbf{m} \in \mathcal{S}_{0,0}^{r}$, one has

$$
\begin{aligned}
&\left(t_{a}^{\alpha}\right)^{-1}\left\langle\boldsymbol{\tau}^{\mathbf{m}+\boldsymbol{\delta}_{a}^{\alpha}} \boldsymbol{\kappa}^{\mathbf{p}}\right\rangle_{\beta}= \\
& \sum_{\substack{\mathbf{p}^{\prime}+\mathbf{p}^{\prime \prime}=\mathbf{p} \\
\mathbf{p}^{\prime \prime} \in \mathcal{S}_{0}^{r}}} \sum_{\nu=o}^{r}\left(\begin{array}{c}
\mathbf{p} \\
\mathbf{p}^{\prime}
\end{array}\right) c_{e\left(\mathbf{p}^{\prime \prime}\right), \alpha}^{\nu} \mathbf{s}^{\mathbf{p}^{\prime \prime}}\left(s_{\left|\mathbf{p}^{\prime \prime}\right|+a-1}^{\nu}\right)^{-1}\left\langle\boldsymbol{\tau}^{\mathbf{m}} \boldsymbol{\kappa}^{\left.\mathbf{p}^{\prime}+\boldsymbol{\delta}_{\left|\mathbf{p}^{\prime \prime}\right|+a-1}^{\nu}\right\rangle_{\beta}} .\right.
\end{aligned}
$$

Here we take $\mathbf{p}^{\prime \prime} \in \mathcal{S}_{0, r}$ since $\pi^{*}\left(\kappa_{-1, \alpha}\right)=\widehat{\kappa}_{-1, \alpha}$. One can also derive a recursion relation in case when $a=0$, and $\mathbf{m}$ is arbitrary, but it is rather messy, and we will not need it in the sequel. The recursion relations (5) are equivalent to the differential equations

$$
\frac{\partial H}{\partial t_{a}^{\alpha}}=\sum_{\mathbf{p} \in \mathcal{S}_{0}^{r}} \sum_{\nu=0}^{r} c_{e(\mathbf{p}), \alpha}^{\nu} \frac{\mathbf{s}^{\mathbf{p}}}{\mathbf{p} !} \frac{\partial H}{\partial s_{|\mathbf{p}|+a-1}^{\nu}}
$$


valid for all $a \geq 1$, and for $a=0$ if instead of $H$ we consider the function $H_{1}$ which is obtained from $H$ by setting all variables $t_{i}^{\mu}$ with $i \geq 1$ to zero.

We want to show that (5) and Prop. 5.3 allow us to compute inductively all intersection numbers of the $\psi$ and the $\kappa$ classes provided we know the Gromov-Witten invariants of $V$. Notice that (5) allows one to eliminate $\tau_{a}^{\alpha}$ with $a \geq 1$ from the intersection numbers. Therefore we reduced the calculation to the case of the intersection numbers of the $\kappa$ classes and the pull backs of the cohomology classes from $V$ if no $\psi$ classes are present.

First we want to reduce everything to the case of the intersection numbers of $\kappa_{-1, \alpha}$ and $e v_{i}^{*}\left(e_{\mu}\right)$. Suppose that we have an intersection number of the type $\left\langle\boldsymbol{\tau}^{\mathbf{m}} \boldsymbol{\kappa}^{\mathbf{p}}\right\rangle_{\beta}$, where $\mathbf{m} \in \mathcal{S}_{0,0}^{r}$, and there exists $p_{a}^{\alpha}>0$ for some $a \geq 0$. If $\|\mathbf{m}\| \geq 2$, then the second or third recursion relation in Prop. 5.3 allows us to express it in terms of the intersection numbers of the type $\left\langle\boldsymbol{\tau}^{\mathbf{m}^{\prime}} \boldsymbol{\kappa}^{\mathbf{p}^{\prime}}\right\rangle_{\beta_{1}}$, where $\mathbf{m}^{\prime} \in \mathcal{S}_{0,0}^{r},\left\|\mathbf{m}^{\prime}\right\| \geq 1$, and either $\left\|\mathbf{p}^{\prime}\right\|<\|\mathbf{p}\|$, or $\left\|\mathbf{p}^{\prime}\right\|=\|\mathbf{p}\|$, and $\left|\mathbf{p}^{\prime}\right|<|\mathbf{p}|$.

However, if $\|\mathbf{m}\|=1$, we can not apply the recursion relations from Prop. 5.3. Here we have to use (5). Namely, pick $\alpha$ such that $\left|e_{\alpha}\right|=2$ and $\int_{\beta} \alpha \neq 0$. (Clearly, such $\alpha$ exists if $V$ is not a point.) When we apply (5) to $\left\langle\boldsymbol{\tau}^{\mathbf{m}+\boldsymbol{\delta}_{0}^{\alpha}} \boldsymbol{\kappa}^{\mathbf{p}}\right\rangle_{\beta}$ we can split the sum in the right hand side into two parts. One term corresponds to $\mathbf{p}^{\prime}=\mathbf{p}$, and $\mathbf{p}^{\prime \prime}=\mathbf{0}$. This term is

$$
\left\langle\boldsymbol{\tau}^{\mathbf{m}} \boldsymbol{\kappa}^{\mathbf{p}} \kappa_{-1, \alpha}\right\rangle_{\beta}=\left\langle\boldsymbol{\tau}^{\mathbf{m}} \boldsymbol{\kappa}^{\mathbf{p}}\right\rangle_{\beta} \int_{\beta} \alpha
$$

Here and in the rest of the argument we disregard the formal variables since they just take care of the signs which do not play an important role in the our argument. The second term is

$$
\sum_{\substack{\mathbf{p}^{\prime}+\mathbf{p}^{\prime \prime}=\mathbf{p} \\
\mathbf{0} \neq \mathbf{p}^{\prime \prime} \in \mathcal{S}_{0}^{r}}} \sum_{\nu=0}^{r}\left(\begin{array}{c}
\mathbf{p} \\
\mathbf{p}^{\prime}
\end{array}\right) c_{e\left(\mathbf{p}^{\prime \prime}\right), \alpha}^{\nu}\left\langle\boldsymbol{\tau}^{\mathbf{m}} \boldsymbol{\kappa}^{\left.\mathbf{p}^{\prime}+\boldsymbol{\delta}_{\left|\mathbf{p}^{\prime \prime}\right|-1}^{\nu}\right\rangle_{\beta} .}\right.
$$

For all these terms

$$
\left\|\mathbf{p}^{\prime}+\boldsymbol{\delta}_{\left|\mathbf{p}^{\prime \prime}\right|-1}^{\nu}\right\|=\left\|\mathbf{p}^{\prime}\right\|+1 \leq\|\mathbf{p}\| .
$$

The equality is attained only when $\mathbf{p}^{\prime \prime}=\boldsymbol{\delta}_{j}^{\mu}$ for $j \geq 1$. But for such terms $\left|\mathbf{p}^{\prime}+\boldsymbol{\delta}_{\left|\mathbf{p}^{\prime \prime}\right|-1}^{\nu}\right|<|\mathbf{p}|$. It follows that we can express each intersection number $\left\langle\boldsymbol{\tau}^{\mathbf{m}} \boldsymbol{\kappa}^{\mathbf{p}}\right\rangle_{\beta}$ with $\mathbf{m} \in \mathcal{S}_{0,0}^{r},\|\mathbf{m}\| \geq 1$ in terms of the intersection numbers $\left\langle\boldsymbol{\tau}^{\mathbf{m}^{\prime}} \boldsymbol{\kappa}^{\mathbf{p}^{\prime}}\right\rangle_{\beta_{1}}$, where either $\left\|\mathbf{p}^{\prime}\right\|<\|\mathbf{p}\|$, or $\left\|\mathbf{p}^{\prime}\right\|=\|\mathbf{p}\|$ and $\left|\mathbf{p}^{\prime}\right|<|\mathbf{p}|$. Note, that it is easy to extend our argument to the case when $\|\mathbf{m}\|=0$. 
Inductively this reduces the computation of the intersection numbers to the integrals of the type

$$
\int_{\overline{\mathcal{M}}_{0, n}(V, \beta)} e v_{1}^{*}\left(\gamma_{1}\right) \ldots e v_{n}^{*}\left(\gamma_{n}\right) \kappa_{-1}\left(\gamma_{n+1}\right) \ldots \kappa_{-1}\left(\gamma_{n+l}\right),
$$

where $\gamma_{i}$ are cohomology classes of $V$. Recall that $\pi^{*}\left(e v_{i}^{*}(\gamma)\right)=e v_{i}^{*}(\gamma)$ and $\pi^{*}\left(\kappa_{-1}(\gamma)\right)=\kappa_{-1}(\gamma)$. Iterating this equality one gets that the intersection number (6) is equal to

$$
\int_{\overline{\mathcal{M}}_{0, n+l}(V, \beta)} e v_{1}^{*}\left(\gamma_{1}\right) \ldots e v_{n}^{*}\left(\gamma_{n}\right) e v_{n+1}^{*}\left(\gamma_{n+1}\right) \ldots e v_{n+l}^{*}\left(\gamma_{n+l}\right) \text {. }
$$

The integral above is the Gromov-Witten invariant evaluated on the classes $\gamma_{1}, \ldots, \gamma_{n+l}$.

Remark. In [27 the authors show how to compute the intersection numbers of the $\psi$ classes and the pull backs of the cohomology classes from $V$ provided that the Gromov-Witten invariants are known. One can show that (5) allows one to eliminate the $\kappa$ classes from the intersection numbers. This provides an alternative way of calculating these intersection numbers via the Gromov-Witten invariants.

\section{REFERENCES}

1. E. Arbarello, M. Cornalba, Combinatorial and algebro-geometric cohomology classes on the moduli space of curves, J. Algebraic Geom. 5 (1996), 705-749.

2. V. V. Batyrev, Stringy Hodge numbers and Virasoro algebra, alg-geom/9711019.

3. K. Behrend, Gromov-Witten invariants in algebraic geometry, Invent. Math. 127 (1997), 601-617.

4. K. Behrend, B. Fantechi, The intrinsic normal cone, Invent. Math. 128 (1997), no. $1,45-88$.

5. K. Behrend, Yu. Manin, Stacks of stable maps and Gromov-Witten invariants. Duke Math. J. 85 (1996), no. 1, 1-60.

6. M. Cornalba, On the projectivity of the moduli spaces of curves, J. Reine Angew. Math. 443, (1993) 11-20.

7. R. Dijkgraaf, Intersection theory, integrable hierarchies and topological field theory, "New Symmetry Principles in Quantum Field Theory," G. Mack Ed. Plenum (1993), 95-158.

8. B. Dubrovin, Geometry of $2 D$ topological field theories, "Integrable systems and Quantum Groups," Lecture Notes in Math. 1620, Springer, Berlin (1996).

9. T. Eguchi, K. Hori, C. S. Xiong, Quantum cohomology and the Virasoro algebra, hep-th/9703086.

10. T. Eguchi, M. Jinzenji, C. S. Xiong, Quantum cohomology and free field representation, hep-th/9709152.

11. W. Fulton, "Intersection Theory," Springer-Verlag Berlin, Heidelberg 1984.

12. W. Fulton, R. Pandharipande, Notes on stable maps and quantum cohomology, alg-geom/9608011. 
13. E. Getzler, Private communication.

14. E. Getzler, M. Kapranov, Modular operads, Preprint, Department of Mathematics, MIT, August 1994, dg-ga/9408003, to appear in Compositio Math.

15. __ Cyclic operads and cyclic homology, to appear in "Geometry, Topology, and Physics for Raoul," (ed. S. T. Yau), International Press, Cambridge, MA, 1994.

16. N. Hitchin, Frobenius manifolds, "Gauge theory and Symplectic Geometry," eds. J. Hurtubise and F. Lalonde, NATO-ASO Series C: 488 1997, Kluwer, Boston.

17. P. Di Francesco, C. Itzykson, Quantum intersection rings, "The moduli space of curves," 81-148, Progr. Math., 129, Birkhaüser, Boston 1995.

18. A. Kabanov, T. Kimura, Intersection numbers and rank one cohomological field theories in genus one, alg-geom/9706003, Max Planck Preprint June 1996, To Appear Comm. Math. Phys.

19. , In preparation.

20. V. G. Kac, A. Schwartz, Geometric interpretation of the partition function of 2D gravity, Phys. Lett. 257 (1991), 329-334.

21. R. Kaufmann, Yu. I. Manin, D. Zagier, Higher Weil-Petersson volumes of moduli spaces of stable n-pointed curves, Commun. Math. Phys. 181 (1996), 763787.

22. S. Keel, Intersection theory of moduli spaces of stable n-pointed curves of genus zero, Trans. AMS 330 (1992), 545-574.

23. F. F. Knudsen, The projectivity of the moduli space of stable curves, II, III, Math. Scandinavica 52 (1983), 161-199, 200-212.

24. J. Kollár, "Rational curves on algebraic varieties," Springer-Verlag, Berlin, 1995.

25. M. Kontsevich, Intersection theory on the moduli space of curves and the matrix Airy function, Commun. Math. Phys. 147 (1992), 1-23.

26. M. Kontsevich, Yu. I. Manin, Gromov-Witten classes, quantum cohomology, and enumerative geometry, Commun. Math. Phys. 164 (1994), 525-562.

27. _ Relations between the correlators of the topological sigma-model coupled to gravity, alg-geom/9708024.

28. M. Kontsevich, Yu. I. Manin (with Appendix by R. Kaufmann), Quantum cohomology of a product, Invent. Math. 124 (1996), 313-340.

29. E. Looijenga, Intersection theory on Deligne-Mumford compactifications [after Witten and Kontsevich], Séminaire Bourbaki, Vol. 1992/93, Astérisque 216 (1993), 187-212.

30. Yu. I. Manin, Frobenius manifolds, quantum cohomology, and moduli spaces (Chapters I, II, III), MPI Preprint No. 96 - 113, January 1996.

31. _ Algebraic geometric introduction to the gravitational quantum cohomology, in preparation.

32. D. Mumford, Towards an enumerative geometry of the moduli space of curves, in "Arithmetic and Geometry," (eds. M. Artin and J. Tate), Part II, Progress in Math., Vol. 36, Birkhäuser, Basel (1983), 271-328.

33. R. Pandharipande, A reconstruction theorem for gravitational descendents, Mittag-Leffler preprint.

34. _ A geometric construction of Getzler's relation, alg-geom/9705016.

35 . , Private communication. 
36. Y. Ruan, G. Tian, A mathematical theory of quantum cohomology, J. Diff. Geom. 42 (1995), 259-367.

37. E. Witten, Two-dimensional gravity and intersection theory on moduli space, Surveys in Diff. Geom. 1 (1991), 243-310.

Max Planck Institut für Mathematik, Gottfried Claren Str. 26, 53225 Bonn, GERMANY And Department of Mathematics, Michigan State University, Wells Hall, East Lansing, MI 48824-1027, USA

E-mail address: kabanov@math.msu.edu

Max Planck Institut für Mathematik, Gottfried Claren Str. 26, 53225 Bonn, GERMANY And Department of Mathematics, 111 CumMington Street, Boston University, Boston, MA 02215, USA

E-mail address: kimura@math.bu.edu 